\title{
\begin{tabular}{l|l} 
Mitraries & DSpace@MIT
\end{tabular}
}

\author{
MIT Open Access Articles
}

Bargaining in Stationary Networks

The MIT Faculty has made this article openly available. Please share how this access benefits you. Your story matters.

Citation: Manea, Mihai. “Bargaining in Stationary Networks.” American Economic Review 101.5 (2011): 2042-2080. Web. (C) 2011 American Economic Association.

As Published: http://dx.doi.org/10.1257/aer.101.5.2042

Publisher: American Economic Association

Persistent URL: http://hdl.handle.net/1721.1/73022

Version: Final published version: final published article, as it appeared in a journal, conference proceedings, or other formally published context

Terms of Use: Article is made available in accordance with the publisher's policy and may be subject to US copyright law. Please refer to the publisher's site for terms of use. 


\title{
Bargaining in Stationary Networks
}

\author{
By Mihai ManeA
}

\begin{abstract}
We study an infinite horizon game in which pairs of players connected in a network are randomly matched to bargain. Players who reach agreement are replaced by new players at the same positions in the network. We show that all equilibria are payoff equivalent. The payoffs and the set of agreement links converge as players become patient. Several new concepts-mutually estranged sets, partners, and shortage ratios-provide insights into the relative strengths of the positions in the network. We develop a procedure to determine the limit equilibrium payoffs for all players. Characterizations of equitable and nondiscriminatory networks are also obtained. (JEL C78, D85)
\end{abstract}

Competitive equilibrium theory assumes large and anonymous markets in which every buyer can trade with every seller. Underlying these assumptions are standard goods and services that may be traded at low transaction costs by agents who are not in specific relationships with one another. However, in many markets goods and services are heterogeneous (e.g., cars, apartments) or need to be tailored to particular needs (e.g., manufacturing inputs, technical support). Furthermore, trading opportunities may depend on transportation costs, social relationships, information, advertising, trust, technological compatibility, joint business opportunities, free trade agreements, etc. In such cases it is natural to model the market using a network, where only pairs of connected agents may engage in exchange.

New theories are needed to explore the influence of the network structure on market outcomes. Many questions arise: How does an agent's position in the network determine his bargaining power and the local prices he faces? Who trades with whom and on what terms? When are prices uniform in the network?

One possible conjecture is that an agent's bargaining power is determined by his (relative) number of connections in the network. However, this simple theory is implausible. Consider the network of four sellers (located at the top nodes) and nine buyers (located at the bottom nodes) depicted in Figure 1. Assume that each seller supplies one unit of a homogeneous indivisible good, each buyer demands one unit of the good, and all buyers have identical values for the good. The buyer located in the middle has the largest number of links in the network, as he is connected to each

\footnotetext{
* Department of Economics, MIT, 50 Memorial Drive, Cambridge, MA 02142 (e-mail: manea@mit.edu). I am grateful to Drew Fudenberg for continual guidance in approaching this project and to Dilip Abreu for valuable perspectives. I thank Jeff Ely, Sambuddha Ghosh, Faruk Gul, John Hatfield, Fuhito Kojima, Michael Ostrovsky, Marcin Peski, Al Roth, Satoru Takahashi, Rakesh Vohra, Asher Wolinsky, and Muhamet Yildiz for helpful comments.

${ }^{\dagger}$ To view additional materials, visit the article page at http://www.aeaweb.org/articles.php?doi=10.1257/aer.101.5.2042.
} 


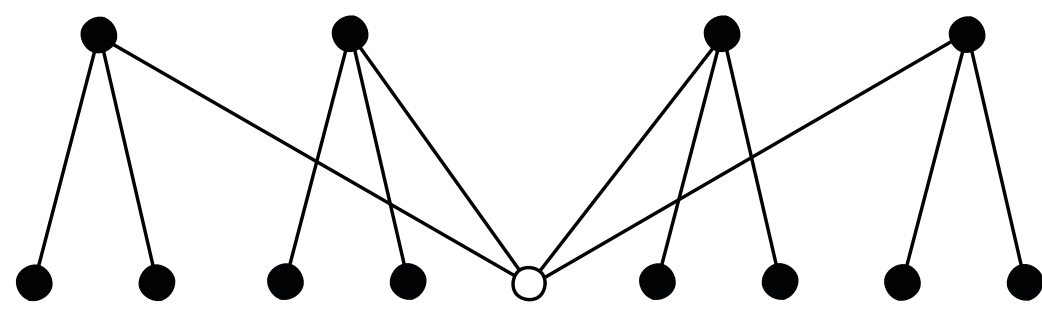

Figure 1. The Position with the Largest Number of Connections Is Not NecESSARILY THE StrongeSt

of the four sellers. Yet every seller has monopoly power over two other buyers whom he can extort, even if trade with the better connected buyer is unattainable. Hence the middle buyer is not able to extract a large fraction of the surplus from any seller despite his relatively large number of connections.

The example above illustrates that the relative strengths of the positions in a network are highly interdependent. An agent's bargaining power does not depend only on the number of his partners, but also on the identities and bargaining power of his partners. Each partner's bargaining power depends in turn on the strengths of his corresponding partners, and so forth. An adequate measure of bargaining power needs to reflect the global network structure.

A recent book by Matthew O. Jackson (2008) surveys the emerging field of social and economic networks and concludes that several central issues remain unsolved.

There are important open questions regarding how network structure affects the distribution of the benefits that accrue to different actors in a network (p. 459).

In particular, Jackson notes that analyzing "a noncooperative game that completely models the bargaining process through which ultimate payoffs are determined [...] is usually intractable" (p. 412). The present paper attempts to fill this gap using a noncooperative model of decentralized bilateral bargaining in networks. Our model is tractable and provides answers to the questions raised earlier.

The benchmark model is as follows. We consider a network where each pair of players connected by a link can jointly produce a unit surplus. The network generates the following infinite horizon discrete time bargaining game. Each period a link is selected according to some probability distribution, and one of the two matched players is randomly chosen to make an offer to the other player specifying a division of the unit surplus between themselves. If the offer is accepted, the two players exit the game with the shares agreed on. We make the following steady state assumption. The two players who reached agreement are replaced in the next period by two new players at the same positions in the network. If the offer is rejected, the two players remain in the game for the next period. All players have a common discount factor.

The steady state assumption captures the idea that in many markets agents face stationary distributions of bargaining opportunities. In such cases, some agents take similar positions in relationships and transactions at different points in time. Our benchmark model provides a stylized depiction of steady states in an economy with multiple populations and a complex pattern of trading opportunities. In the multiple 
population interpretation, each node corresponds to a population of identical agents, and the network represents the structure of interactions among populations. From this perspective, our setting constitutes an extension of Ariel Rubinstein and Asher Wolinsky's (1985) fundamental model of bargaining in stationary markets with two populations.

Indeed, the results carry over to a model in the spirit of Douglas M. Gale's (1987), where every period a continuum of players is present at each node in the network, and a positive measure of player pairs are matched to bargain across each link. While in the benchmark model the replacement assumption entails that every period an inflow of agents matches the stochastic endogenous outflow of agents reaching agreements in equilibrium, in the multiple population version the steady state analysis involves a deterministic inflow. This enables us to investigate how the size of the population at each node is determined in a steady state of an economy with exogenously specified inflows of agents into the network. Manea (2010) studies a more general model of multipopulation bargaining with an emphasis on nonstationary markets.

Depending on the application, populations may correspond to buyers and sellers of used cars with specific parameters (brand, mileage, manufacturing year, fuel efficiency, warranty, etc.); renters with specific demands (in terms of location, number of bedrooms, recent renovation, dishwasher availability, etc.) and the corresponding landlords; firms with different types of production technologies and workers with particular training and job preferences; importers and exporters who trade goods that meet certain quality standards. In the former two applications, populations can be further divided according to exposure to various advertising platforms, demographics, and locations of buyers and sellers. In the latter, trading companies are differentiated by the ability to meet the requirements of particular markets, trade permits, and business connections.

In general, a population is defined by a category of agents who share the same relevant attributes for the market under consideration. The network of exchange is determined by the compatibility of the factors describing various populations. Background elements such as word-of-mouth communication, consumer protection laws, transportation costs, and trade agreements also shape the network. For example, a prospective renter is effectively connected only to landlords who meet his apartment search criteria and whom he finds through acquaintances, real estate agencies, or advertisements. In markets with multiple populations, the steady state assumption translates into the stationarity of the distribution of buyer and seller types.

Suppose that in the underlying network a certain group of buyers can trade only with a relatively small number of sellers. One might surmise that these sellers can use their oligopoly power to capture a significant fraction of the gains from trade. This is not necessarily the case for each seller involved. Due to network asymmetries, the sellers may have unequal bargaining power. Indeed, some subsets of the original oligopoly may face higher demand and exert more market power relative to others. For instance, if a subset of the sellers is connected to only one of the buyers, then the buyer can exploit his monopsony power. Some of the remaining sellers will form an oligopoly with greater market power than initially assumed. Other delicate issues arise. For example, overlapping oligopolies may cater to a common customer base or split up into several smaller oligopolies with varying degrees of bargaining power. 
The set of oligopolies and the price distribution emerging in equilibrium depend on the network structure in a complex fashion. The partition of the market into oligopolies and the relative strengths of the oligopolies are determined endogenously via decentralized bargaining. Our analysis reveals that the most powerful oligopolies - the ones with the lowest seller-to-buyer ratio-drive market outcomes. Roughly speaking, for patient agents, the network is decomposed into a series of oligopolies, which are ordered decreasingly according to market power. Each oligopoly in the list includes all buyers and sellers with extremal payoffs among those not involved in the preceding oligopolies.

\section{Outline of the Results}

We assume that the network structure is common knowledge and all players have perfect information about the events preceding any of their decision nodes in the game. The equilibrium concept we use is subgame perfect equilibrium. [1

In Section III, we show that for every discount factor the equilibrium payoff of every player present in the game at the beginning of any period is uniquely determined by his position in the network (Theorem 1). For all but a finite number of discount factors, there exists a partition of the set of links into equilibrium agreement and disagreement links (Proposition 1). In every equilibrium, after any history, a pair of players connected by an equilibrium agreement link reaches an agreement when matched to bargain, and the terms of the agreement are uniquely pinned down by the positions in the network of the proposer and the responder. Players connected by disagreement links never reach agreement. We prove that there exists a limit equilibrium agreement network that describes the set of agreement links for sufficiently high discount factors. Also, the equilibrium payoffs converge as players become patient (Theorem 2).

For an illustration, consider the bargaining game on the five-player network $G_{1}$ shown in Figure 2, where all links are selected for bargaining with equal probability. For every discount factor there is a unique equilibrium, with agreement network $G_{1}$. In equilibrium every match ends in agreement because players 4 and 5 cannot be monopolized by either player 1 or 2 . The limit equilibrium payoffs are $3 / 5$ for players 1 and 2 , and $2 / 5$ for players 3,4 , and 5. The limit equilibrium agreement network coincides with $G_{1}$.

Consider next the game induced by the network $G_{2}$, which is obtained from $G_{1}$ by removing the link $(2,4)$. We assume again that every link is drawn for bargaining with the same probability. For low discount factors there exists a unique equilibrium, with the agreement network given by $G_{2}$. However, for high discount factors, players 1 and 5 do not reach an agreement in equilibrium when matched to bargain. The intuition is that player 1 can extort players 3 and 4 , since these two players do not have other bargaining partners. Player 1 cannot extract as much surplus from player 5 , since 5 has monopoly over player 2 . The limit equilibrium payoffs are

\footnotetext{
${ }^{1}$ Section III discusses the robustness of the results to some features of the information structure and equilibrium requirements.

${ }^{2}$ In all figures, limit equilibrium payoffs for each player are represented next to the corresponding node, and limit equilibrium agreement and disagreement links are drawn as thick and thin line segments, respectively.
} 

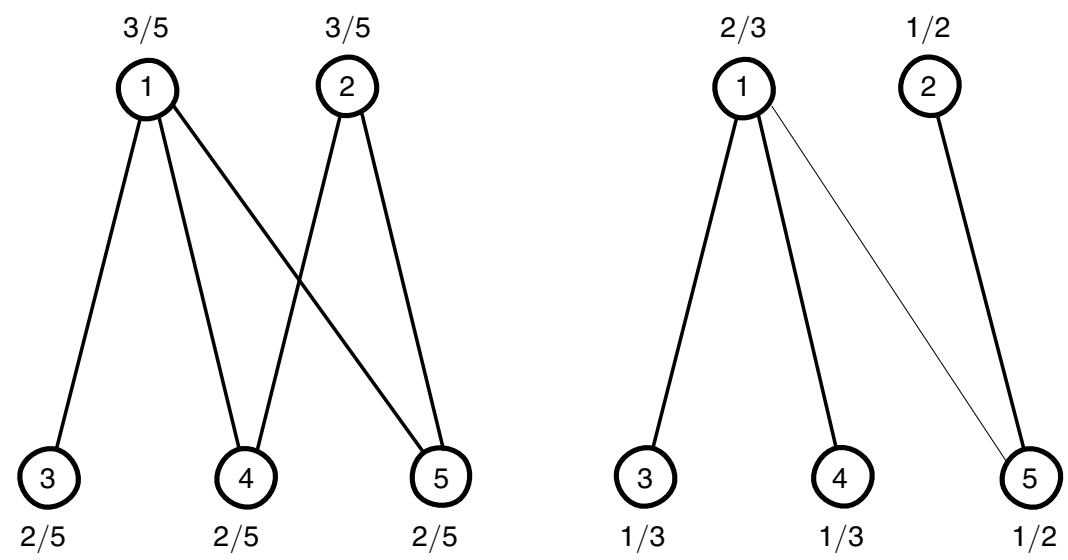

Figure 2. Networks $G_{1}\left(\right.$ LEFT) AND $G_{2}$

$2 / 3$ for player $1,1 / 3$ for players 3 and 4 , and $1 / 2$ for players 2 and 5 . The limit equilibrium agreement network consists of all links of $G_{2}$ except $(1,5)$. The equilibria of the bargaining games on the networks $G_{1}$ and $G_{2}$ for all discount factors are described in detail in Example 1 from Section III.

The main objective is to characterize the limit equilibrium payoffs for every network. The following essential observation is presented in Section IV. Consider a set of players who are pairwise disconnected in the limit equilibrium agreement network and the set of players with whom these players share limit agreement links. We refer to players in the former set as mutually estranged and to ones in the latter as partners. As players become patient, the partners have control over the (equilibrium) relevant bargaining opportunities of the mutually estranged players. For high discount factors, since the estranged players can reach agreements only in equilibrium with the partners, the mutually estranged set is weak if the partners are relatively scarce. The appropriate measure of the strength of a mutually estranged set proves to be the simplest that springs to mind-the shortage ratio, which is defined as the ratio of the number of partners to estranged players.

For example, in the network $G_{1}$ the shortage ratios of the mutually estranged sets $\{3,4\}$ and $\{3,4,5\}$ are 1 and $2 / 3$, respectively, since the partner set is $\{1,2\}$ in either case. In the network $G_{2}$ the shortage ratios of the mutually estranged sets $\{3,4\}$ and $\{3,4,5\}$ are $1 / 2$ and $2 / 3$, respectively, since the corresponding partner sets are $\{1\}$ and $\{1,2\}$, respectively. The determination of the partners for the mutually estranged sets considered here is based on the aforementioned limit equilibrium agreement subnetworks for the bargaining games on the networks $G_{1}$ and $G_{2}$.

The concepts of mutually estranged sets, partners, and shortage ratios play a key role in the prediction of bargaining power. Formally, the shortage ratio measures the strength of a mutually estranged set in the following sense. For every set of mutually estranged players and their partners, the ratio of the limit equilibrium payoffs of the worst-off estranged player and the best-off partner is not larger than the shortage ratio of the mutually estranged set (Theorem 3). The result yields an upper (lower) bound for the limit payoff of the worst-off estranged player (best-off partner).

There may be a multitude of mutually estranged sets, and it is not immediately clear which, if any, of the corresponding bounds for the limit equilibrium payoffs 
are binding. One delicate step toward the main result (Theorem 4) is the idea that the bounds generated by a set of mutually estranged players and their partners need to be binding unless the worst-off estranged player is part of an even weaker mutually estranged set, and the best-off partner is part of an even stronger partner set. Based on this intuition, we prove that the extreme bounds - the ones derived from the (largest) mutually estranged set that minimizes the shortage ratio and the corresponding partners - must bind. The two sets of players associated with these bounds have extremal limit equilibrium payoffs and induce an oligopoly subnetwork enclosing all their limit agreement links. Thus, for high discount factors, the partners act as an oligopoly that corners and extorts the estranged players. In the equilibrium limit, surplus within the oligopoly subnetwork is divided according to the shortage ratio of the mutually estranged players with respect to their partners, with all players on each side receiving identical payoffs.

Section $\mathrm{V}$ develops an algorithm that sequentially determines the limit equilibrium payoffs of all players based on the ideas above. At each step, the algorithm determines the union of all mutually estranged sets with the lowest shortage ratio and removes the corresponding estranged players and their partners. Within the identified extremal oligopoly subnetwork surplus is divided between the two sides according to the shortage ratio. The algorithm stops when all players have been removed or when the lowest shortage ratio is greater than or equal to 1 . The latter scenario corresponds to limit payoffs of $1 / 2$ for the remaining players.

The limit equilibrium payoffs for the network $G_{2}$ are obtained by computing the lowest shortage ratio to be $1 / 2$, attained for the mutually estranged set $\{3,4\}$ with the partner set $\{1\}$. Then the limit payoffs are $2 / 3$ for player 1 and $1 / 3$ for players 3 and 4 . Once we remove players 1,3 , and 4 , the lowest shortage ratio in the remaining network is 1 ; hence, players 2 and 5 obtain limit payoffs of $1 / 2$.

We can use the algorithm to investigate the uniformity of payoffs in a network. A network is called equitable if it does not create differentiated bargaining power as players become patient; that is, all players have limit equilibrium payoffs of $1 / 2$. In Section VI we show that a network is equitable if and only if it is quasi-regularizable; another equivalent condition is that the network have an edge cover which is the disjoint union of a matching and odd cycles (Theorem 5).

Section VII restricts attention to buyer-seller networks and provides a more straightforward characterization of the equilibrium limit (Theorem $4^{B S}$ ). Shortage ratios smaller than 1 and limit payoffs of $1 / 2$ do not play a special role in the results for buyer-seller networks. We also analyze nondiscriminatory buyer-seller networks, where the limit payoffs of all buyers are identical. If the buyer-seller ratio is an integer, then the network is nondiscriminatory if and only if it can be covered by a disjoint union of clusters formed by one seller connected to a number of buyers equal to the buyer-seller ratio (Theorem 6).

In Section VIII, we clarify the replacement assumption in the context of a market where there is a continuum of agents every period. Each node corresponds to a population of identical agents, and the network represents the pattern of exchange

\footnotetext{
${ }^{3}$ Our analysis reveals that the lowest shortage ratio, when smaller than 1 , may be computed by considering sets of pairwise disconnected players, along with their neighbors, in the original network rather than in the (a priori unknown) limit equilibrium agreement network.
} 
opportunities between populations. In the analog stationary game for the multiple population setting, a constant measure of players is present at each node of the network in every period. The matching technology is such that, for each link in the network, a mass of pairs of players at the two endpoints is selected for bargaining. The steady state assumption delivering the stationary structure of the game is that the set of players from a given node who reach agreements is immediately replaced by an equal measure of new players at that node. Under this assumption, the steady state analysis of the benchmark model extends without difficulty.

The characterization of the equilibrium payoffs and agreements in the stationary game can be used to address the issue of determining a steady state when the set of potential market entrants is exogenously specified. Theorem 7 is a corollary of an existence result established in the framework of Manea (2010). Suppose that there is a constant stream of potential entrants at each node in the network. For any matching technology that varies continuously with the stationary distribution of agents in the market, there exist small entry costs for each node such that the resulting economy has a steady state. Manea (2010) shows that strengthening this result to apply for every set of small entry costs is not possible.

Rubinstein and Wolinsky (1985) and Gale (1987) analyze markets in steady state abstracting away from existence issues and invoking replacement assumptions analogous to ours. Similarly, the focus here is on the equilibrium outcomes of bargaining in stationary markets, rather than the mechanics of steady states. While it is useful to close the model with exogenous inflows as in the setting of Section VIII, the key contribution of this paper is the characterization of bargaining power in stationary networks developed in Sections II through VII.

Section IX reviews the related literature, and Section $\mathrm{X}$ concludes. An online Appendix discusses network stability with respect to the limit equilibrium payoffs, extensions of the results to heterogenous discount factors, and an example in which the bargaining protocol is asymmetric.

\section{Framework}

Let $N$ denote the set of $n$ players, $N=\{1,2, \ldots, n\}$. A network is an undirected graph $H=(V, E)$ with set of vertices $V \subset N$ and set of edges-also called links$E \subset\{(i, j) \mid i \neq j \in V\}$ such that $(j, i) \in E$ whenever $(i, j) \in E$. We identify the pairs $(i, j)$ and $(j, i)$, and use the shorthand $i j$ or $j i$ instead. We say that player $i$ is connected in $H$ to player $j$, or $i$ has an $H$ link to $j$, if $i j \in E$. We regularly abuse notation and write $i j \in H$ for $i j \in E$. A network $H^{\prime}=\left(V^{\prime}, E^{\prime}\right)$ is a subnetwork of $H$ if $V^{\prime} \subset V$ and $E^{\prime} \subset E$. A network $H^{\prime}=\left(V^{\prime}, E^{\prime}\right)$ is the subnetwork of $H$ induced by $V^{\prime}$ if $E^{\prime}=$ $E \cap\left(V^{\prime} \times V^{\prime}\right)$.

Fix a network $G$ with vertex set $N$. Let $\left(p_{i j}>0\right)_{i j \in G}$ be a matching technology for $G$, which is a probability distribution over $G$ 's links. A link $i j$ in $G$ is interpreted

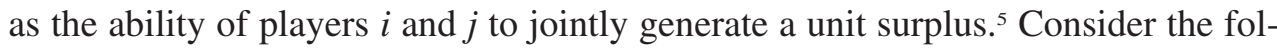
lowing infinite horizon bargaining game generated by the network $G$. Each period $t=0,1, \ldots$ a link $i j$ in $G$ is selected with probability $p_{i j}$, and one of the players (the

\footnotetext{
${ }^{4}$ Throughout the paper, $\subset$ denotes weak inclusion; other authors prefer the symbol $\subseteq$.

${ }^{5}$ Without loss of generality, we assume that each player has at least one link in $G$.
} 
proposer) $i$ and $j$ is chosen randomly (with equal probability) to make an offer to the other player (the responder) specifying a division of the unit surplus between themselves. If the responder accepts the offer, the two players exit the game with the shares agreed upon. In period $t+1$ two new players assume the same positions in the network as $i$ and $j$, respectively. If the responder rejects the offer, the two players remain in the game for the next period. In period $t+1$ the game is played in the same manner with the set of $n$ players, consisting of the ones from period $t$, with the departing players replaced by new players if an agreement obtains in period $t$. All players share a discount factor $\delta \in(0,1)$. The game is denoted $\Gamma^{\delta}$.

Formally, there exists a sequence $i_{0}, i_{1}, \ldots, i_{\tau}, \ldots$ of players of type $i \in N$ (a player's type is defined by his position in the network). When player $i_{\tau}$ exits the game (following an agreement with another player), player $i_{\tau+1}$ replaces him the next period. All players have common knowledge of the game, including the network structure and the matching technology. We assume that players have perfect information of all the events preceding any of their decision nodes in the game. Possible relaxations of the informational assumptions regarding past bargaining outcomes are discussed in the next section.

There are three types of histories. We denote by $h_{t}$ a history of the game up to (not including) time $t$, which is a sequence of $t$ pairs of proposers and responders connected in $G$, with corresponding proposals and responses. We call such histories, and the subgames that follow them, complete. For simplicity, we assume that for every history players are labeled only by their type without reference to the index of their copy. The index $\tau$ of the player of type $i$ playing the game at time $t$ following the history $h_{t}$ can be recovered by counting the number of bargaining agreements involving $i$ along $h_{t}$. Therefore, for each $i \in N$, a history $h_{t}$ uniquely determines the player $i_{\tau}$ present in the game at time $t$, and when there is no risk of confusion we suppress the index $\tau$. We denote by $\mathcal{H}\left(i_{\tau}\right)$ the set of complete histories, or subgames, where $i_{\tau}$ is the player of type $i$ present in the game. We denote by $\left(h_{t} ; i \rightarrow j\right)$ the history consisting of $h_{t}$ followed by nature selecting $i$ to propose to $j$. We denote by $\left(h_{t} ; i \rightarrow j ; x\right)$ the history consisting of $\left(h_{t} ; i \rightarrow j\right)$ followed by $i$ offering $x \in[0,1]$ to $j$.

A strategy $\sigma_{i_{\tau}}$ for player $i_{\tau}$ specifies, for all $j$ connected to $i$ in $G$ and all $h_{t} \in$ $\mathcal{H}\left(i_{\tau}\right)$, the offer $\sigma_{i_{\tau}}\left(h_{t} ; i \rightarrow j\right)$ that $i$ makes to $j$ following the history $\left(h_{t} ; i \rightarrow j\right)$, and the response $\sigma_{i_{\tau}}\left(h_{t} ; j \rightarrow i ; x\right)$ that $i$ gives to $j$ after the history $\left(h_{t} ; j \rightarrow i ; x\right)$. We allow for mixed strategies, hence $\sigma_{i_{\tau}}\left(h_{t} ; i \rightarrow j\right)$ and $\sigma_{i_{\tau}}\left(h_{t} ; j \rightarrow i ; x\right)$ are probability distributions over $[0,1]$ and $\{$ Yes, No\}, respectively. In the context of our game, we say that two strategy profiles are payoff equivalent if they induce identical payoffs for any player $i_{\tau}$, where $i_{\tau}$ 's payoff is evaluated as the expected value of his gains from all the agreements discounted relative to his date of entry into the game (which is not period 0 for $\tau \geq 1$ ). A strategy profile $\left(\sigma_{i_{\tau}}\right)_{i \in N, \tau \geq 0}$ is a subgame perfect equilibrium of $\Gamma^{\delta}$ if it induces Nash equilibria in subgames following every history $\left(h_{t} ; i \rightarrow j\right)$ and $\left(h_{t} ; i \rightarrow j ; x\right)$.

\footnotetext{
${ }^{6}$ The results extend to an alternative specification of the model where every period there is a continuum of players at every node and a positive mass of players is matched to bargain across each link. Section VIII develops this approach.
} 


\section{Essential Equilibrium Uniqueness and Discounting Asymptotics}

We first show that across all equilibria of the bargaining game, the expected payoff of every player present in any complete subgame is uniquely determined by his position in the network. The unique and stationary equilibrium payoffs associated with each player type may be used to describe the possible equilibrium outcomes of every bargaining encounter.

THEOREM 1: For every $\delta \in(0,1)$, there exists a payoff vector $\left(v_{i}^{* \delta}\right)_{i \in N}$ such that for every subgame perfect equilibrium of $\Gamma^{\delta}$ the expected payoff of player $i_{\tau}$ in any $\mathcal{H}\left(i_{\tau}\right)$ subgame is uniquely given by $v_{i}^{* \delta}$ for all $i \in N, \tau \geq 0$. For every equilibrium of $\Gamma^{\delta}$, in any subgame where $i_{\tau}$ is selected to make an offer to $j_{\tau^{\prime}}$, the following statements hold with probability one:

(i) if $\delta\left(v_{i}^{* \delta}+v_{j}^{* \delta}\right)<1$ then $i_{\tau}$ offers $\delta v_{j}^{* \delta}$ and $j_{\tau^{\prime}}$ accepts;

(ii) if $\delta\left(v_{i}^{* \delta}+v_{j}^{* \delta}\right)>1$ then $i_{\tau}$ makes an offer that $j_{\tau^{\prime}}$ rejects.

We can extend the conclusions of Theorem 1 to settings in which players do not have perfect information about all past matchings but still have common knowledge of the network structure. It may be that players know only their own history of interactions or know the history of all pairs matched to bargain but see the outcomes of only their own interactions. Players who reach agreements and exit the game may pass down information to the players who take their positions in the network. A player may learn that his bargaining partner has type $i$ but be uncertain whether he is dealing with player $i_{0}, i_{1}, \ldots$ In such settings, players need to form beliefs about the unrevealed bargaining outcomes. Extending the proof to show uniqueness of the sequential equilibrium payoffs for each player type under various information structures is straightforward. The uniqueness argument relies solely on the assumption that in every match the proposer knows the position of the responder in the (commonly known) network. ${ }^{\text {] }}$

Manea (2010) establishes equilibrium payoff equivalence in markets with multiple populations where the probability that any two player types are matched at a given date is exogenous. Theorem 1 is a special case of that uniqueness result, and we omit its proof here. However, we present a partial argument showing that all stationary equilibria in the current model are payoff equivalent. The ideas of the argument also play an important role in subsequent results.

A strategy profile $\left(\sigma_{i_{\tau}}\right)_{i \in N, \tau \geq 0}$ is stationary if each player's strategy at any time $t$ depends exclusively on his position in the network and the play of the game in period $t$, that is, $\sigma_{i_{\tau}}\left(h_{t} ; i \rightarrow j\right)=\sigma_{i_{\tau^{\prime}}}\left(h_{t^{\prime}}^{\prime} ; i \rightarrow j\right)$ and $\sigma_{i_{\tau}}\left(h_{t} ; j \rightarrow i ; x\right)=\sigma_{i_{\tau^{\prime}}}\left(h_{t^{\prime}}^{\prime} ; j \rightarrow i ; x\right)$ for all $i j \in G, x \in[0,1], \tau, \tau^{\prime} \geq 0, h_{t} \in \mathcal{H}\left(i_{\tau}\right), h_{t^{\prime}}^{\prime} \in \mathcal{H}\left(i_{\tau^{\prime}}\right)$. A stationary equilibrium is a subgame perfect equilibrium in stationary strategies.

Let $\sigma$ be a stationary equilibrium of $\Gamma^{\delta}$. Under $\sigma$, for every $i \in N$, each player $i_{\tau}$ receives the same expected payoff, $\tilde{v}_{i}$, in any $\mathcal{H}\left(i_{\tau}\right)$ subgame. Suppose that $i j \in G$

\footnotetext{
${ }^{7}$ Theorem 1 generalizes to payoff equivalence of security equilibria (K. G. Binmore and M. J. Herrero 1988b).
} 
and $i$ is selected to propose to $j$ in a subgame. Since $j$ 's continuation payoff in case of disagreement is $\delta \tilde{v}_{j}$, in equilibrium player $j$ accepts any offer larger than $\delta \tilde{v}_{j}$ and rejects offers smaller than $\delta \tilde{v}_{j}$.

If $\delta \tilde{v}_{i}<1-\delta \tilde{v}_{j}, i$ offers $\delta \tilde{v}_{j}$ and $j$ accepts with probability 1 . Indeed, any offer $x<\delta \tilde{v}_{j}$ is rejected, leading to a continuation payoff of $\delta \tilde{v}_{i}<1-\delta \tilde{v}_{j}$ for $i$, while any offer $x>\delta \tilde{v}_{j}$ is accepted, leaving $i$ with a payoff of $1-x$. In equilibrium, it must be that $i$ offers $x=\delta \tilde{v}_{j}$ and $j$ accepts with probability 1$]$ Similarly, if $\delta \tilde{v}_{i}>1-\delta \tilde{v}_{j}$, $i$ makes an offer that $j$ rejects with probability 1 . Making any proposal that is acceptable to $j$ would leave $i$ with at most $1-\delta \tilde{v}_{j}$, which is less than his continuation payoff of $\delta \tilde{v}_{i}$. When $\delta \tilde{v}_{i}=1-\delta \tilde{v}_{j}, i$ is indifferent between offering $j$ the minimum $\delta \tilde{v}_{j}$ necessary for an agreement and making an unacceptable offer. In all cases, $j$ obtains a payoff of $\delta \tilde{v}_{j}$ regardless of whether he accepts the offer or the game proceeds to the next round without agreement.

The claims above hold for every $i j \in G$. It follows that, for every $i \in N$, in any subgame following the selection of a link and a proposer, player $i$ obtains a payoff different from $\delta \tilde{v}_{i}$ only if he was selected to make an offer to a player $j$ with $\delta \tilde{v}_{i}<1-\delta \tilde{v}_{j}$. The latter event occurs with probability $p_{i j} / 2$ and leads to a payoff of $1-\delta \tilde{v}_{j}$ for $i$. These observations are succinctly captured by the following equation:

$$
\tilde{v}_{i}=\left(1-\sum_{\{j \mid i j \in G\}} \frac{p_{i j}}{2}\right) \delta \tilde{v}_{i}+\sum_{\{j \mid i j \in G\}} \frac{p_{i j}}{2} \max \left(1-\delta \tilde{v}_{j}, \delta \tilde{v}_{i}\right) .
$$

Hence, $\tilde{v}$ is a fixed point of the function $f^{\delta}=\left(f_{1}^{\delta}, f_{2}^{\delta}, \ldots, f_{n}^{\delta}\right):[0,1]^{n} \rightarrow[0,1]^{n}$ defined by

$$
f_{i}^{\delta}(v)=\left(1-\sum_{\{j \mid i j \in G\}} \frac{p_{i j}}{2}\right) \delta v_{i}+\sum_{\{j \mid i j \in G\}} \frac{p_{i j}}{2} \max \left(1-\delta v_{j}, \delta v_{i}\right) .
$$

Lemma 6 in the Appendix establishes that $f^{\delta}$ is a contraction with respect to the sup norm on $\mathbb{R}^{n}$. Hence $f^{\delta}$ has a unique fixed point, denoted $v^{* \delta}$. Since the stationary equilibrium payoffs of $\Gamma^{\delta}$ need to be fixed points of $f^{\delta}$, they are uniquely given by $v^{*}$.

REMARK 1: The set of pure strategy stationary equilibria of $\Gamma^{\delta}$ is nonempty. Indeed, the following strategies constitute an equilibrium. When $i$ is selected to propose to $j$, he offers $\min \left(1-\delta v_{i}^{* \delta}, \delta v_{j}^{* \delta}\right)$, and when $i$ has to respond-regardless of the proposer-he accepts any offer of at least $\delta v_{i}^{* \delta}$ and rejects smaller offers. However, the payoff equivalence of stationary equilibria does not imply uniqueness of the equilibrium strategies. For instance, when $i$ is selected to make an offer to $j$ and $\delta\left(v_{i}^{* \delta}+v_{j}^{* \delta}\right)>1$, we can replace $i$ 's behavior in the equilibrium construction above by any mixed strategy over the interval $\left[0, \delta v_{j}^{* \delta}\right.$ ) or $\left[0, \delta v_{j}^{* \delta}\right]$ (depending on whether $j$ 's strategy is to accept offers of $\delta v_{j}^{* \delta}$ from $i$ with positive probability).

\footnotetext{
${ }^{8}$ As in standard bargaining arguments, an offer $x>\delta \tilde{v}_{j}$ cannot arise in equilibrium because $i$ would have incentives to deviate to any offer $x^{\prime} \in\left(\delta \tilde{v}_{j}, x\right)$. If $j$ does not accept $\delta \tilde{v}_{j}$ with probability 1 , then $i$ has no best response to j's strategy.
} 
The following description of the equilibria for two simple networks illustrates the conclusions of Theorem 1. Equilibria for more complex networks are analyzed in Example 2. The uniform matching technology for the network $G$, deployed in Examples 1-3, is defined by $p_{i j}=1 / e, \forall i j \in G$ where $e$ denotes the total number of links in $G$.

Example 1: Consider the network $G_{1}$ illustrated in Figure 2. The equilibrium payoffs in the game $\Gamma^{\delta}$ played on the network $G_{1}$, with the uniform matching technology, are

$$
\begin{aligned}
& v_{1}^{* \delta}=\frac{150-250 \delta+103 \delta^{2}}{5\left(100-220 \delta+158 \delta^{2}-37 \delta^{3}\right)} \\
& v_{2}^{* \delta}=\frac{100-160 \delta+63 \delta^{2}}{5\left(100-220 \delta+158 \delta^{2}-37 \delta^{3}\right)} \\
& v_{3}^{* \delta}=\frac{2\left(25-40 \delta+16 \delta^{2}\right)}{5\left(100-220 \delta+158 \delta^{2}-37 \delta^{3}\right)} \\
& v_{4}^{* \delta}=v_{5}^{* \delta}=\frac{100-165 \delta+67 \delta^{2}}{5\left(100-220 \delta+158 \delta^{2}-37 \delta^{3}\right)},
\end{aligned}
$$

converging to $v_{1}^{*}=v_{2}^{*}=3 / 5$ and $v_{3}^{*}=v_{4}^{*}=v_{5}^{*}=2 / 5$ as $\delta \rightarrow 1$. There exists a unique equilibrium in which, for all $i j \in G_{1}$, when $i$ is selected to propose to $j$, he offers $\delta v_{j}^{*}$, and when $i$ has to respond to a proposal from $j$, he accepts any offer of at least $\delta v_{i}^{*} \delta$ and rejects smaller offers. In equilibrium every match results in agreement.

Consider next the network $G_{2}$, also illustrated in Figure 2. When players have discount factor $\delta \leq 10(9-\sqrt{2}) / 79 \approx 0.9602=: \underline{\delta}$, the equilibrium payoffs in the bargaining game on the network $G_{2}$, with the uniform matching technology, are

$$
\begin{aligned}
& v_{1}^{* \delta}=\frac{300-520 \delta+223 \delta^{2}}{5\left(200-460 \delta+346 \delta^{2}-85 \delta^{3}\right)} \\
& v_{2}^{* \delta}=\frac{100-160 \delta+63 \delta^{2}}{5\left(200-460 \delta+346 \delta^{2}-85 \delta^{3}\right)} \\
& v_{3}^{* \delta}=v_{4}^{* \delta}=\frac{2\left(50-85 \delta+36 \delta^{2}\right)}{5\left(200-460 \delta+346 \delta^{2}-85 \delta^{3}\right)} \\
& v_{5}^{* \delta}=\frac{2\left(100-170 \delta+71 \delta^{2}\right)}{5\left(200-460 \delta+346 \delta^{2}-85 \delta^{3}\right)} .
\end{aligned}
$$

For $\delta \leq \underline{\delta}$, there is a unique equilibrium with a description similar to the case of $G_{1}$.

A payoff irrelevant equilibrium multiplicity arises for the discount factor $\underline{\delta}$. For $\delta=\underline{\delta}$, it is true that $\delta\left(v_{1}^{* \delta}+v_{5}^{* \delta}\right)=1$. Any behavior of player 1 in interactions with player 5 satisfying the following conditions is part of an equilibrium. Player 1's offer 
is an arbitrary probability distribution over $\left[0, \delta v_{5}^{* \delta}\right]$. Player 1 rejects offers smaller than $\delta v_{1}^{* \delta}$, accepts with some arbitrary probability an offer of $\delta v_{1}^{* \delta}$, and accepts with probability 1 larger offers. ${ }^{\text {. }}$

The equilibrium payoffs when $\delta>\underline{\delta}$ are

$$
v_{1}^{* \delta}=\frac{2}{10-7 \delta}, v_{3}^{* \delta}=v_{4}^{* \delta}=\frac{1}{10-7 \delta}, v_{2}^{* \delta}=v_{5}^{* \delta}=\frac{1}{2(5-4 \delta)},
$$

converging to $v_{1}^{*}=2 / 3, v_{3}^{*}=v_{4}^{*}=1 / 3$, and $v_{2}^{*}=v_{5}^{*}=1 / 2$ as $\delta \rightarrow 1$. For $\delta>\underline{\delta}$, in every equilibrium agreement obtains across all links except $(1,5)$. The equilibrium requirements do not pin down the disagreement offer in an encounter between players 1 and 5, and there exist multiple payoff equivalent equilibria as explained in Remark 1. However, in every equilibrium, the strategies for bargaining across the links $(1,3),(1,4),(2,5)$ must be as specified in Remark 1.

We call $\left(v_{i}^{*}\right)_{i \in N}$ the equilibrium payoff vector at $\delta$. The equilibrium agreement network at $\delta$, denoted $G^{*}$, is defined as the subnetwork of $G$ with the link $i j$ included if and only if $\delta\left(v_{i}^{* \delta}+v_{j}^{* \delta}\right) \leq 1$. Hence the equilibrium agreement network consists of all links where the two players at the endpoints can come up with a division of the surplus that makes both weakly better off than proceeding without agreement to the next period. For $\delta$ such that $\delta\left(v_{i}^{* \delta}+v_{j}^{* \delta}\right) \neq 1, \forall i j \in G$, the agreements and disagreements in any subgame across all equilibria are entirely characterized as in the second part of Theorem 1 . Across a link $i j \in G^{* \delta}$, if $\delta\left(v_{i}^{* \delta}+v_{j}^{* \delta}\right)<1$ then agreement is reached with probability 1 in any equilibrium; if $\delta\left(v_{i}^{* \delta}+v_{j}^{* \delta}\right)=1$ then there exist equilibria in which agreement is reached with any probability. For links $i j \notin G^{*} \delta$, disagreement arises with probability 1 in any equilibrium. We show that the condition $\delta\left(v_{i}^{* \delta}+v_{j}^{* \delta}\right) \neq 1, \forall i j \in G$ holds for all but a finite set of discount factors $\delta$, hence the description of equilibrium agreements and disagreements provided by Theorem 1 is complete for generic discount factors.

PROPOSITION 1: The condition $\delta\left(v_{i}^{* \delta}+v_{j}^{* \delta}\right) \neq 1, \forall i j \in G$ holds for all but a finite set of $\delta$.

The proof appears in the Appendix. We outline the approach here since some of its elements resurface in the proof of the next result. For every $\delta \in(0,1)$ and every subnetwork $H$ of $G$, consider the $n \times n$ system of linear equations

$$
v_{i}=\left(1-\sum_{\{j \mid i j \in H\}} \frac{p_{i j}}{2}\right) \delta v_{i}+\sum_{\{j \mid i j \in H\}} \frac{p_{i j}}{2}\left(1-\delta v_{j}\right), \forall i=\overline{1, n} .
$$

\footnotetext{
${ }^{9}$ Note that the probability of agreement between $1_{\tau}$ and $5_{\tau^{\prime}}$ does not influence their own payoffs, but affects the length of time that future players of types 1 and 5 need to wait before entering the game. However, the equilibrium payoffs of future players are not affected by the induced delay since, as already mentioned, a player's payoff is evaluated by discounting relative to his entry into the game rather than period 0 .
} 
We have shown above that $v^{* \delta}$ solves the system for $H=G^{* \delta}$. It is easy to check that the system (3) has a unique solution $v^{\delta, H}$. In particular, $v^{* \delta}=v^{\delta, G^{* \delta}}$.

All entries in the augmented matrix of the linear system (3) are linear functions of $\delta$. Then for each $i \in N$, the solution $v_{i}^{\delta, H}$ is given by Cramer's rule, as the ratio of two determinants that are polynomials in $\delta$ of degree at most $n$,

$$
v_{i}^{\delta, H}=P_{i}^{H}(\delta) / Q_{i}^{H}(\delta)
$$

We can then argue that every $\delta$ for which there exist $i j \in G$ with $\delta\left(v_{i}^{* \delta}+v_{j}^{* \delta}\right)=1$ is a root of one of a finite family of nonzero polynomials in $\delta$.

Denote by $\Delta$ the finite set of $\delta$ for which there exists $i j \in G$ with $\delta\left(v_{i}^{* \delta}+v_{j}^{* \delta}\right)=1$. As established by Theorem 1 , for $\delta \notin \Delta$, in every equilibrium of $\Gamma^{\delta}$, in any subgame where $i_{\tau}$ is chosen to make an offer to $j_{\tau^{\prime}}$, with probability one: (1) if $i j \in G^{* \delta}$ then $i_{\tau}$ offers $\delta v_{j}^{* \delta}$ and $j_{\tau^{\prime}}$ accepts; (2) if $i j \notin G^{* \delta}$ then $i_{\tau}$ makes an offer that $j_{\tau^{\prime}}$ rejects.

The following result establishes convergence of the equilibrium outcomes as players become patient. The proof appears in the Appendix.

THEOREM 2: There exist $\underline{\delta} \in(0,1)$ and a subnetwork $G^{*}$ of $G$ such that the equilibrium agreement network $G^{* \delta}$ is equal to $G^{*}$ for all $\delta>\underline{\delta}$. The equilibrium payoff vector $v^{* \delta}$ converges to a vector $v^{*}$ as $\delta$ goes to 1 . The rate of convergence of $v^{* \delta}$ to $v^{*}$ is $O(1-\delta)$.

We call $G^{*}$ the limit equilibrium agreement network and $v^{*}$ the limit equilibrium payoff vector. Our main objective is to determine the limit equilibrium payoffs. ${ }^{10}$ The following preliminary observations are proven in the Appendix.

PROPOSITION 2: If $i j \in G$, then $v_{i}^{*}+v_{j}^{*} \geq 1$. If $i j \in G^{*}$, then $v_{i}^{*}+v_{j}^{*}=1$. In particular, if $v_{i}^{*}+v_{j}^{*}>1$, then ij $\notin G^{*}$.

LEMMA 1: Every player has at least one link in $G^{*}$ (under the assumption in footnote 5).

REMARK 2: The benchmark model assumes that all agreements generate the same surplus. The bargaining protocol specifies that in every match both players are equally likely to be chosen as the proposer. However, the results of this section generalize without difficulty to a setting with heterogenous link values and arbitrary asymmetric probabilities of recognizing the proposer conditional on every link selection.

\footnotetext{
${ }^{10}$ One can approximate the equilibrium payoffs for a given discount factor $\delta$ by iterating the contraction $f^{\delta}$ on any initial payoff vector until convergence to its fixed point is obtained. Note that the contraction factor is $\delta$. This computational approach can be used to determine the payoffs in any given network. However, we seek to provide a conceptual characterization of bargaining power in arbitrary networks.
} 


\section{Bounds for Limit Equilibrium Payoffs}

In order to characterize the limit equilibrium payoffs, we need to introduce several new concepts. For every network $H$ and subset of vertices $M$, let $L^{H}(M)$ denote the set of players who have $H$-links to players in $M, L^{H}(M)=\{j \mid i j \in H, i \in M\}$. A set $M$ is $H$-independent if there exists no $H$-link between two players in $M, M \cap$ $L^{H}(M)=\varnothing$. A nonempty set of players is mutually estranged if it is $G^{*}$-independent. The set of partners for a mutually estranged set $M$ is defined as $L^{G^{*}}(M)$. [1]

Fix a mutually estranged set $M$ with partner set $L$. Basically, as players become patient, the set $L$ spans the relevant bargaining opportunities of the set $M$. For high discount factors, since the players in $M$ can reach equilibrium agreements only when matched to bargain with players in $L$, the set $M$ is weak if $L$ is relatively small. This intuition is formalized by the shortage ratio of $M$, defined as the ratio of the number of partners to estranged players, $|L| /|M|$. The shortage ratio measures the collective strength of the mutually estranged players in a sense made precise by Theorems 3 and 4.

The next result is essential for developing a procedure to determine the limit equilibrium payoffs. For every mutually estranged set $M$ with partner set $L$, the ratio of the limit equilibrium payoffs of the worst-off estranged player, $\min _{i \in M} v_{i}^{*}$, and the best-off partner, $\max _{j \in L} v_{j}^{*}$, is not larger than the shortage ratio of $M$.

THEOREM 3: For every mutually estranged set $M$ with partner set $L$, the following bounds on limit equilibrium payoffs hold:

$$
\begin{gathered}
\min _{i \in M} v_{i}^{*} \leq \frac{|L|}{|M|+|L|} \\
\max _{j \in L} v_{j}^{*} \geq \frac{|M|}{|M|+|L|} .
\end{gathered}
$$

The proof of Theorem 3 is relegated to the Appendix. It uses the following result, which follows immediately from rearranging equation (1) (with the substitution $\left.\tilde{v}=v^{* \delta}\right)$.

LEMMA 2: For every $\delta$, the equilibrium payoff of each player in $\Gamma^{\delta}$ is equal to the expected present value of his stream of first mover advantage, i.e.,

$$
v_{i}^{* \delta}=\frac{1}{1-\delta} \sum_{\{j \mid i j \in G\}} \frac{p_{i j}}{2} \max \left(1-\delta v_{i}^{* \delta}-\delta v_{j}^{* \delta}, 0\right), \forall i \in N, \forall \delta \in(0,1) .
$$

We say that $\max \left(1-\delta v_{i}^{* \delta}-\delta v_{j}^{* \delta}, 0\right)$ measures the first mover advantage that $i$ gains from making an offer to $j$. Indeed, as argued in Section III, the expected pay-

\footnotetext{
${ }^{11}$ To illustrate the definitions, note that the list of all mutually estranged sets and corresponding partner sets in the limit equilibrium agreement network for the bargaining game on the network $G_{2}$ (Figure 2$)$ is $(\{1\},\{3,4\})$, $(\{2\},\{5\}),(\{3\},\{1\}),(\{4\},\{1\}),(\{5\},\{2\}),(\{1,2\},\{3,4,5\}),(\{1,5\},\{2,3,4\}),(\{2,3\},\{1,5\}),(\{2,4\},\{1,5\}),(\{3,4\}$, $\{1\}),(\{3,5\},\{1,2\}),(\{4,5\},\{1,2\}),(\{2,3,4\},\{1,5\})$, and $(\{3,4,5\},\{1,2\})$.
} 
off of $i$ is $\delta v_{i}^{* \delta}$ in any subgame following nature's move where he is not the proposer and $\max \left(1-\delta v_{j}^{* \delta}, \delta v_{i}^{* \delta}\right)$ in any subgame where he is selected to make an offer to player $j$. Hence player $i$ 's net benefit from getting the opportunity to make an offer to $j$ is the difference $\max \left(1-\delta v_{j}^{* \delta}, \delta v_{i}^{* \delta}\right)-\delta v_{i}^{* \delta}=\max \left(1-\delta v_{i}^{* \delta}-\delta v_{j}^{* \delta}, 0\right)$.

The intuition for the proof of Theorem 3 is as follows. Suppose that $M$ is a mutually estranged set with partner set $L$. Fix a discount factor $\delta>\underline{\delta}$, with $\underline{\delta}$ specified as in Theorem 2. Thus, $G^{* \delta}=G^{*}$. In every equilibrium, in any subgame, each player $i$ in $M$ reaches agreements only with players in $L$ with whom he shares $G^{*}$ links. Any first mover advantage that a player $i$ in $M$ gains from making an offer to a player $j$ in $L$ is mapped to an equal first mover advantage that $j$ gains from making an offer to $i\left(\max \left(1-\delta v_{i}^{* \delta}-\delta v_{j}^{*}, 0\right)\right.$ is symmetric in $i$ and $\left.j\right)$. Moreover, both gains are weighted by the same probability, $p_{i j} / 2$, in the expected payoffs of $i$ and $j$ since the two players are equally likely to make offers when matched to bargain. It follows that the sum of the expected present values of the streams of first mover advantage enjoyed by all players in $M$ is not larger than the same expression evaluated for the players in $L .12$ Hence, by Lemma $2, \sum_{j \in L} v_{j}^{* \delta} \geq \sum_{i \in M} v_{i}^{* \delta}$. Note that the symmetry of the bargaining protocol is essential for this conclusion. Taking the limit $\delta \rightarrow 1$ we obtain that $\sum_{j \in L} v_{j}^{*} \geq \sum_{i \in M} v_{i}^{*}$. Then the proof is made complete by repeatedly using Proposition 2 to establish that $\min _{i \in M} v_{i}^{*}+\max _{j \in L} v_{j}^{*}=1$.

While Theorem 3 invokes knowledge we do not have a priori about the limit equilibrium agreement network $G^{*}$, it has an immediate corollary that exclusively involves properties of $G .{ }^{13}$ It is sufficient to note that since $G^{*}$ is a subnetwork of $G$, if $M$ is $G$-independent then $M$ is also $G^{*}$-independent, and $L^{G^{*}}(M) \subset L^{G}(M)$, so $\left|L^{G^{*}}(M)\right| \leq\left|L^{G}(M)\right|$.

COROLLARY 1: For every $G$-independent set of players $M$, the following bounds on limit equilibrium payoffs hold:

$$
\begin{aligned}
\min _{i \in M} v_{i}^{*} & \leq \frac{\left|L^{G}(M)\right|}{|M|+\left|L^{G}(M)\right|} \\
\max _{j \in L^{G}(M)} v_{j}^{*} & \geq \frac{|M|}{|M|+\left|L^{G}(M)\right|} .
\end{aligned}
$$

\section{Limit Equilibrium Payoff Computation}

Theorem 3 suggests that it may be useful to study the mutually estranged sets $M$ that minimize the upper bound $\left|L^{G^{*}}(M)\right| /\left(|M|+\left|L^{G^{*}}(M)\right|\right)$ for the limit equilibrium payoff of the worst-off player in $M$ or, equivalently, minimize the shortage ratio $\left|L^{G^{*}}(M)\right| /|M|$. The next lemma-applied to the network $G^{*}$-shows that the set of such minimizers is closed with respect to unions if the attained minimum is less than 1. It is useful to generalize this conclusion to all networks. The proof is in the

\footnotetext{
${ }^{12}$ Players in $M$ gain first mover advantage only from players in $L$, while players in $L$ gain first mover advantage from the corresponding players in $M$, and possibly from players outside $M$.

${ }^{13}$ However, for the results of the next section we need the full strength of Theorem 3.
} 
Appendix. For every network $H$, let $\mathcal{I}(H)$ denote the set of nonempty $H$-independent sets.

LEMMA 3: Let $H$ be a network. Suppose that

$$
\min _{M \in \mathcal{I}(H)} \frac{\left|L^{H}(M)\right|}{|M|}<1,
$$

and that $M^{\prime}$ and $M^{\prime \prime}$ are two H-independent sets achieving the minimum. Then $M^{\prime} \cup M^{\prime \prime}$ is also H-independent and

$$
M^{\prime} \cup M^{\prime \prime} \in \underset{M \in \mathcal{I}(H)}{\arg \min } \frac{\left|L^{H}(M)\right|}{|M|} .
$$

We show that the bounds on limit equilibrium payoffs corresponding to a set of mutually estranged players and their partners provided by Theorem 3 need to be binding unless the worst-off estranged player is part of an even weaker mutually estranged set, and the best-off partner is part of an even stronger partner set. The intuition is that each player is part of a limit equilibrium oligopoly subnetwork where, as $\delta \rightarrow 1$, some mutually estranged players and their partners share the unit surplus according to the shortage ratio. Consequently, the limit payoff of any player cannot be smaller than the upper bound for the worst-off player from a mutually estranged set with the lowest shortage ratio. Therefore, the bounds for the limit equilibrium payoffs of the worst-off estranged player and the best-off partner corresponding to a mutually estranged set with the lowest shortage ratio must be binding.

Suppose that the lowest shortage ratio, $r_{1}=\min _{M \in \mathcal{I}(G)}\left|L^{G}(M)\right| /|M|$, is smaller than 1 . Let $M_{1}$ be the union of all $G$-independent sets $M$ minimizing the shortage ratio. By Lemma $3, M_{1}$ is also a $G$-independent set with minimal shortage ratio. Let $L_{1}=L^{G}\left(M_{1}\right)$ be the corresponding set of partners.

REMARK 3: Note that $G^{*}$ is a priori unknown. Our analysis is self-contained in that it does not directly involve $G^{*}$. Many steps in the proof of Theorem 4 below uncover properties of $G^{*}$ that enable the application of Theorem 3. In particular, we show that the lowest shortage ratio-when smaller than 1-may be computed by restricting attention to sets that are $G$-independent rather than $G^{*}$-independent. Another key finding is that $L^{G^{*}}\left(M_{1}\right)=L^{G}\left(M_{1}\right)$. Therefore, we legitimately refer to $M_{1}$ as the largest mutually estranged set minimizing the shortage ratio, to $L_{1}$ as the partner set of $M_{1}$, and to $r_{1}$ as the shortage ratio of $M_{1}$.

We have argued above that $\min _{i \in M_{1}} v_{i}^{*}=r_{1} /\left(r_{1}+1\right)$ and $\max _{j \in L_{1}} v_{j}^{*}=1 /\left(r_{1}+1\right)$. We set out to show that the limit equilibrium payoffs are given by $r_{1} /\left(r_{1}+1\right)$ for all players in $M_{1}$ and $1 /\left(r_{1}+1\right)$ for all players in $L_{1}$. That is, all players in $M_{1} \cup L_{1}$, not only the worst-off estranged player and the best-off partner, have extremal limit payoffs. The following algorithm sequentially iterates this hypothesis in order to determine the limit equilibrium payoffs of all players. 
DEFINITION 1: (Algorithm $\left.\mathcal{A}(G)=\left(r_{s}, x_{s}, M_{s}, L_{s}, N_{s}, G_{s}\right)_{s=1,2, \ldots, \bar{s}}\right)$ Define the sequence $\left(r_{s}, x_{s}, M_{s}, L_{s}, N_{s}, G_{s}\right)_{s}$ recursively as follows: Let $N_{1}=N$ and $G_{1}=G$. For $s \geq 1$, if $N_{s}=\varnothing$ then stop. Otherwise, let ${ }^{[4]}$

$$
r_{s}=\min _{M \subset N_{s}, M \in \mathcal{I}(G)} \frac{\left|L^{G_{s}}(M)\right|}{|M|} .
$$

If $r_{s} \geq 1$ then stop. Else, set $x_{s}=r_{s} /\left(1+r_{s}\right)$. Let $M_{s}$ be the union of all minimizers $M$ in (4). ${ }^{15}$ Denote $L_{s}=L^{G_{s}}\left(M_{s}\right)$. Let $N_{s+1}=N_{s} \backslash\left(M_{s} \cup L_{s}\right)$ and $G_{s+1}$ be the subnetwork of $G$ induced by the players in $N_{s+1}$. Denote by $\bar{s}$ the finite step at which the algorithm ends. 16

At each step, the algorithm $\mathcal{A}(G)$ determines the largest mutually estranged set minimizing the shortage ratio in the subnetwork induced by the remaining players (Lemma 3), and removes the corresponding estranged players and partners. Remark 3 is essential to the applicability of the procedure. The definition ensures that $\mathcal{A}(G)$ simultaneously identifies and removes all residual players with extremal limit payoffs. Then, for high discount factors, the removed players reach agreements only among themselves in equilibrium, and the network induced by the remaining players can be analyzed independently. The algorithm terminates when all players have been removed or every $G$-independent set formed by the remaining players has shortage ratio greater than or equal to 1 .

As an illustration, the algorithm $\mathcal{A}\left(G_{2}\right)$, for the network $G_{2}$ from the introduction, ends in $\bar{s}=2$ steps. The relevant outcomes are $r_{1}=1 / 2, x_{1}=1 / 3, M_{1}=\{3,4\}$, $L_{1}=\{1\}$ at the first step and $r_{2}=1, N_{2}=\{2,5\}$ at the second.

The next lemma, which is used in the proofs of Proposition 3 and Theorem 4 below, follows immediately from the specification of the algorithm $\mathcal{A}(G)$. The proof of Proposition 3 is provided in the Appendix.

LEMMA 4: $\mathcal{A}(G)$ satisfies the following conditions for all $1 \leq s \leq s^{\prime}<\bar{s}$ :

$$
\begin{gathered}
L^{G_{s}}\left(M_{s} \cup M_{s+1} \cup \ldots \cup M_{s^{\prime}}\right)=L_{s} \cup L_{s+1} \cup \ldots \cup L_{s^{\prime}} \\
L^{G\left(N_{s+1}\right)} \cap\left(M_{1} \cup M_{2} \cup \ldots \cup M_{s}\right)=\varnothing \\
M_{s} \cup M_{s+1} \cup \ldots \cup M_{s^{\prime}} \text { is G-independent. }
\end{gathered}
$$

PROPOSITION 3: The sequences $\left(r_{s}\right)_{s}$ and $\left(x_{s}\right)_{s}$ defined by $\mathcal{A}(G)$ are strictly increasing.

Note that the sets $M_{1}, L_{1}, \ldots, M_{\bar{s}-1}, L_{\bar{s}-1}, N_{\bar{s}}$ partition $N$. The limit equilibrium payoff of each player is uniquely determined by the partition set that includes him.

\footnotetext{
${ }^{14}$ It can be shown that each player in $N_{s}$ has at least one link in $G_{s}$, hence $r_{s}$ is well defined and positive.

${ }^{15}$ By Lemma 3, since $r_{s}<1, M_{s}$ is also a minimizer in (4).

${ }^{16}$ In some cases the last step variables $r_{\bar{s}}, x_{\bar{s}}, M_{\bar{s}}, L_{\bar{s}}$ are unnecessary and are left undefined.
} 
THEOREM 4: Let $\left.\left(r_{s}, x_{s}, M_{s}, L_{s}, N_{s}, G_{s}\right)_{s=1,2, \ldots, \bar{s}}\right)$ be the outcome of the algorithm $\mathcal{A}(G)$. The limit equilibrium payoffs for $\Gamma^{\delta}$ as $\delta \rightarrow 1$ are given by

$$
\begin{aligned}
& v_{i}^{*}=x_{s}, \forall i \in M_{s}, \forall s<\bar{s} \\
& v_{j}^{*}=1-x_{s}, \forall j \in L_{s}, \forall s<\bar{s} \\
& v_{k}^{*}=\frac{1}{2}, \forall k \in N_{\bar{s} .}
\end{aligned}
$$

PROOF:

We prove the theorem by induction on $s$. Suppose we established the assertion for all lower values, and we proceed to proving it for $s(1 \leq s \leq \bar{s}) .17$ We treat the case $s=\bar{s}$ separately in the Appendix.

Let $s<\bar{s}$ and define $\underline{x}_{s}=\min _{i \in N_{s}} v_{i}^{*}$. Denote by $\underline{M}_{s}=\arg \min _{i \in N_{s}} v_{i}^{*}$ the set of players in $N_{s}$ whose limit equilibrium payoffs equal $\underline{x}_{s}$ and set $\underline{L}_{s}=L^{G_{s}}\left(\underline{M}_{s}\right)$. We first show that $\underline{x}_{s}=x_{s}$ by arguing that $\underline{x}_{s} \leq x_{s}$ and $\underline{x}_{s} \geq x_{s}$.

CLAIM 4.1: $\underline{x}_{s} \leq x_{s}$

We proceed by contradiction. Suppose that $\underline{x}_{s}>x_{s}$. Then $v_{j}^{*} \geq 1-x_{s-1}>1-$ $x_{s}>1-\underline{x}_{s}$ for all $j$ in $L_{1} \cup L_{2} \cup \ldots \cup L_{s-1} \cdot \frac{18}{18}$ The first inequality follows from the induction hypothesis and Proposition 3, and the second from Proposition 3. But $v_{i}^{*} \geq \underline{x}_{s}$ for all $i$ in $M_{s}$. Thus, $v_{i}^{*}+v_{j}^{*}>1, \forall i \in M_{s}, \forall j \in L_{1} \cup L_{2} \cup \ldots \cup L_{s-1}$. By Proposition 2 no player $i \in M_{s}$ has $G^{*}$ links to players $j \in L_{1} \cup L_{2} \cup \ldots \cup L_{s-1}$, or $L^{G^{*}}\left(M_{s}\right) \cap\left(L_{1} \cup L_{2} \cup \ldots \cup L_{s-1}\right)=\varnothing$.

By Lemma $4, L^{G}\left(M_{s}\right) \cap\left(M_{1} \cup M_{2} \cup \ldots \cup M_{s-1}\right)=\varnothing$.

It follows that $L^{G^{*}}\left(M_{s}\right) \subset L^{G_{s}}\left(M_{s}\right)=L_{s}$. Theorem 3 implies that

$$
\min _{i \in M_{s}} v_{i}^{*} \leq \frac{\left|L_{s}\right|}{\left|M_{s}\right|+\left|L_{s}\right|}=x_{s},
$$

a contradiction with $\min _{i \in N_{s}} v_{i}^{*}=\underline{x}_{s}>x_{s}$.

CLAIM 4.2: $\underline{x}_{s} \geq x_{s}$ and $v_{j}^{*}=1-\underline{x}_{s}, \forall j \in \underline{L}_{s}$.

We proved that $\underline{x}_{s} \leq x_{s}$. Since $r_{s}<1$ it follows that $x_{s}<1 / 2$. By Proposition 2 and Claim 4.1,

$$
v_{j}^{*} \geq 1-\underline{x}_{s} \geq 1-x_{s}>1 / 2, \forall j \in \underline{L}_{s} .
$$

Then Proposition 2 implies that $\underline{L}_{s}$ is a $G^{*}$-independent set.

\footnotetext{
${ }^{17}$ The following technical detail is used in order to avoid analogous arguments proving the base case and the inductive step. Append step 0 to the algorithm, with $\left(r_{0}, x_{0}, M_{0}, L_{0}, N_{0}, G_{0}\right)=(0,0, \varnothing, \varnothing, N, G)$. Then the base case $s=0$ follows trivially, and the inductive steps, $s=1,2, \ldots, \bar{s}-1$, involve analogous arguments.

${ }^{18}$ This argument is necessary only for $s>1$.
} 
Fix $j \in \underline{L}_{s}$. By Proposition 2 there exist no $G^{*}$ links from $j$ to players $k \in N_{s} \backslash \underline{M}_{s}$, since for these players $v_{k}^{*}>x_{s}$ (by the definition of $\underline{M}_{s}$ ) and we already argued that $v_{j}^{*} \geq 1-\underline{x}_{s}$. Also, there exist no $G$ links from $j$ to players in $M_{1} \cup M_{2} \cup \ldots \cup M_{s-1}$ by Lemma 4.

By Proposition 2, there exist no $G^{*}$ links from $j$ to players $k \in L_{1} \cup L_{2} \cup \ldots \cup L_{s-1}$ since for these players $v_{k}^{*} \geq 1-x_{s-1}>1 / 2$, and we need $v_{j}^{*}>1 / 2$.

Therefore, any $j \in \underline{L}_{s}$ only has $G^{*}$ links to players in $\underline{M}_{s}$. By Proposition 2 and Lemma 1 , any player $j \in \underline{L}_{s}$ must have limit equilibrium payoff $v_{j}^{*}=1-\underline{x}_{s}$.

Hence $\underline{L}_{s}$ is $G^{*}$-independent and $L^{G^{*}}\left(\underline{L}_{s}\right) \subset \underline{M}_{s}$, , 19 so it follows from Theorem 3 that

$$
\underline{x}_{s}=\max _{i \in L^{G^{*}}\left(\underline{L}_{s}\right)} v_{i}^{*} \geq \frac{\left|\underline{L}_{s}\right|}{\left|L^{G^{*}}\left(\underline{L}_{s}\right)\right|+\left|\underline{L}_{s}\right|} \geq \frac{\left|\underline{L}_{s}\right|}{\left|\underline{M}_{s}\right|+\left|\underline{L}_{s}\right|} .
$$

Recall that $\underline{L}_{s}=L^{G_{s}}\left(\underline{M}_{s}\right)$. We can rewrite the inequality above as

$$
\frac{\underline{x}_{s}}{1-\underline{x}_{s}} \geq \frac{\left|L^{G_{s}}\left(\underline{M}_{s}\right)\right|}{\left|\underline{M}_{s}\right|} .
$$

Yet by the definition of $r_{s}$ and $x_{s}$,

$$
\frac{\left|L^{G_{s}}\left(\underline{M}_{s}\right)\right|}{\left|\underline{M}_{s}\right|} \geq r_{s}=\frac{x_{s}}{1-x_{s}}
$$

and the last two inequalities imply that $\underline{x}_{s} \geq x_{s}$.

Claims 4.1 and 4.2 establish that $\underline{x}_{s}=x_{s}$. Hence, $v_{i}^{*}=x_{s}, \forall i \in \underline{M}_{s}$ and $v_{j}^{*}=1-x_{s}$, $\forall j \in \underline{L}_{s}$. Moreover, we need to have equalities in the weak inequalities (5) and (6), so $\left|\underline{L}_{s}\right| /\left|\underline{M}_{s}\right|=r_{s}$.

CLAIM 4.3: $\underline{M}_{s} \subset M_{s}$

Since $M_{s}$ is the union of all $G$-independent $M \subset N_{s}$ with $\left|L^{G_{s}}(M)\right| /|M|=r_{s}$ and $\left|L^{G_{s}}\left(\underline{M}_{s}\right)\right| /\left|\underline{M}_{s}\right|=r_{s}$, it follows that $\underline{M}_{s} \subset M_{s}$. $\underline{M}_{s}$ is $G$-independent by Proposition 2, as the limit equilibrium payoff of each player in $\underline{M}_{s}$ is $x_{s}<1 / 2$.)

CLAIM 4.4: $\underline{M}_{s}=M_{s}$

We show that $M_{s}=M_{s}$ by contradiction. Fix $i \in M_{s} \backslash M_{s}$. Since $i \in M_{s}$ and $L^{G_{s}}\left(M_{s}\right)=L_{s}, i$ has no $G$ links to players in $N_{s} \backslash L_{s}$. By Lemma 4, $i$ has no $G$ links to $M_{1} \cup M_{2} \cup \ldots \cup M_{s-1}$.

By Proposition 2, $i$ has no $G^{*}$ links to players $j \in L_{1} \cup L_{2} \cup \ldots \cup L_{s-1} \cup \underline{L}_{s}$ as for such players $v_{j}^{*} \geq 1-x_{s}$, and $v_{i}^{*}>\underline{x}_{s}=x_{s}$ by the definition of $\underline{M}_{s}$.

It follows that $i$ may only have $G^{*}$ links to players in $L_{s} \backslash L_{s}$. Therefore, $L^{G^{*}}\left(M_{s} \backslash \underline{M}_{s}\right) \subset L_{s} \backslash \underline{L}_{s}$, implying that $\left|L^{G^{*}}\left(M_{s} \backslash \underline{M}_{s}\right)\right| \leq\left|L_{s} \backslash \underline{L}_{s}\right|=\left|L_{s}\right|-\left|\underline{L}_{s}\right|$.

\footnotetext{
${ }^{19}$ It can be easily argued that the inclusion holds with equality.
} 
Note that

$$
\frac{\left|L_{s}\right|}{\left|M_{s}\right|}=r_{s} \& \frac{\left|\underline{L}_{s}\right|}{\left|\underline{M}_{s}\right|}=r_{s} \Rightarrow \frac{\left|L_{s}\right|-\left|\underline{L}_{s}\right|}{\left|M_{s}\right|-\left|\underline{M}_{s}\right|}=r_{s} .
$$

Then by Theorem 3,

$$
\begin{aligned}
\min _{i \in M_{s} \backslash \underline{M}_{s}} v_{i}^{*} & \leq \frac{\left|L^{G^{*}}\left(M_{s} \backslash \underline{M}_{s}\right)\right|}{\left|M_{s} \backslash \underline{M}_{s}\right|+\left|L^{G^{*}}\left(M_{s} \backslash \underline{M}_{s}\right)\right|} \\
& \leq \frac{\left|L_{s}\right|-\left|\underline{L}_{s}\right|}{\left|M_{s}\right|-\left|\underline{M}_{s}\right|+\left|L_{s}\right|-\left|\underline{L}_{s}\right|}=\frac{r_{s}}{1+r_{s}}=x_{s},
\end{aligned}
$$

a contradiction with $v_{i}^{*}>x_{s}$ for all $i \in N_{s} \backslash \underline{M}_{s}$.

Therefore, $\underline{M}_{s}=M_{s}, \underline{L}_{s}=L_{s}$, and $v_{i}^{*}=x_{s}, \forall i \in M_{s}$, and $v_{j}^{*}=1-x_{s}, \forall j \in L_{s}$, completing the proof of the induction step for $s<\bar{s}$.

REMARK 4: The limit equilibrium payoffs are independent of the relative probabilities of links being selected for bargaining $\left(p_{i j}>0\right)_{i j \in G}$.

An important implication of Theorem 4 is that submarkets endogenously emerge in equilibrium. An economy described by a connected network which cannot be decomposed into nonoverlapping submarkets may induce a disconnected limit equilibrium agreement network where agents are partitioned into oligopoly subnetworks. In the equilibrium limit, each oligopoly subnetwork describes a separate submarket since no transactions occur across distinct oligopoly subnetworks. The limit equilibrium prices are uniform within every submarket. Each agent self-selects into the most favorable submarket to which he has access. Although no equilibrium trades occur across different submarkets, the submarkets should not be analyzed independently since the market partition is determined by the underlying network structure.

Example 2 below shows that the limit equilibrium agreement network does not necessarily contain all the links from players in $M_{s}$ to players in $L_{s}$. Although all players in $M_{s}\left(L_{s}\right)$ have identical limit equilibrium payoffs, their relative bargaining strengths may vary, and the rates of convergence to the common limit are not identical across $M_{s}\left(L_{s}\right)$. Moreover, it is possible that the players in $M_{s} \cup L_{s}$ induce a connected subnetwork in $G$, but a disconnected one in $G^{*}$.

Example 2: Consider the bargaining game on the nine-player network $G_{3}$ illustrated in Figure 3, with the uniform matching technology. ${ }^{20}$ The algorithm $\mathcal{A}\left(G_{3}\right)$ terminates in one step, with $r_{1}=1 / 2, M_{1}=\{4,5,6,7,8,9\}$, and $L_{1}=\{1,2,3\}$. Therefore, the limit equilibrium payoffs are $1 / 3$ for all players in $M_{1}$ and $2 / 3$ for all players in 


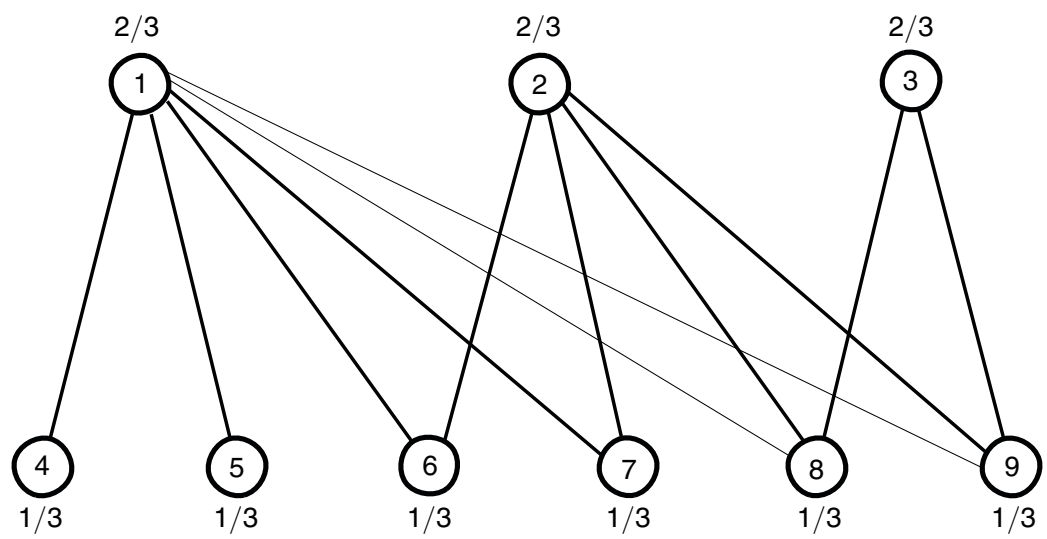

Figure 3. Network $G_{3}$

$L_{1}$. However, it is not the case that the limit equilibrium agreement network contains all the links from players in $M_{1}$ to players in $L_{1}$ (that is, all the links of $G_{3}$ ).

Indeed, the limit equilibrium agreement network $G_{3}^{*}$ excludes the links $(1,8)$ and $(1,9)$. The intuition is that, although $v_{1}^{*}=v_{2}^{*}=v_{3}^{*}=2 / 3$ and $v_{4}^{*}=v_{5}^{*}=v_{6}^{*}=v_{7}^{*}=$ $v_{8}^{*}=v_{9}^{*}=1 / 3$, player 1 is relatively stronger than players 2 and 3 as he is connected to all players that 2 and 3 are connected to, and players 8 and 9 are relatively stronger than players $4,5,6$, and 7 as they are connected to all players with whom $4,5,6$, and 7 are connected. For similar reasons, player 3 is relatively weaker than players 1 and 2, augmenting the relative strength of 8 and 9 over 4, 5,6, and 7; and players 4 and 5 are relatively weaker than players $6,7,8,9$, augmenting the relative strength of 1 over 2 and 3 . For high $\delta$, the equilibrium payoffs of player 1 , and also of players 8 and 9 , will be sufficiently high so that 1 does not reach agreement with either 8 or 9 .

By the proof of Theorem 1, to check that $G_{3}^{*}$ is the limit equilibrium agreement network, we need to show only that $v^{\delta, G_{3}^{*}}$ is the fixed point of the corresponding $f^{\delta}$ for $\delta$ sufficiently large. \The payoff vector $v^{\delta, G_{3}^{*}}$ solves the $9 \times 9$ system of linear equations

$$
v_{i}=\frac{2 e-e_{i}}{2 e} \delta v_{i}+\frac{1}{2 e} \sum_{\left\{j|j| j \in G_{3}^{*}\right\}}\left(1-\delta v_{j}\right), \forall i=\overline{1,9},
$$

where $e$ denotes the total number of links in $G_{3}$ and $e_{i}$ the number of links player $i$ has in $G_{3}^{*}$. A closed-form solution is immediately obtained, but is omitted for expositional brevity. For example,

$$
v_{1}^{\delta, G_{3}^{*}}=\frac{2\left(576-1068 \delta+493 \delta^{2}\right)}{3\left(2304-6048 \delta+5264 \delta^{2}-1519 \delta^{3}\right)},
$$

and the other components of $v^{\delta, G_{3}^{*}}$ have similar rational function expressions. 


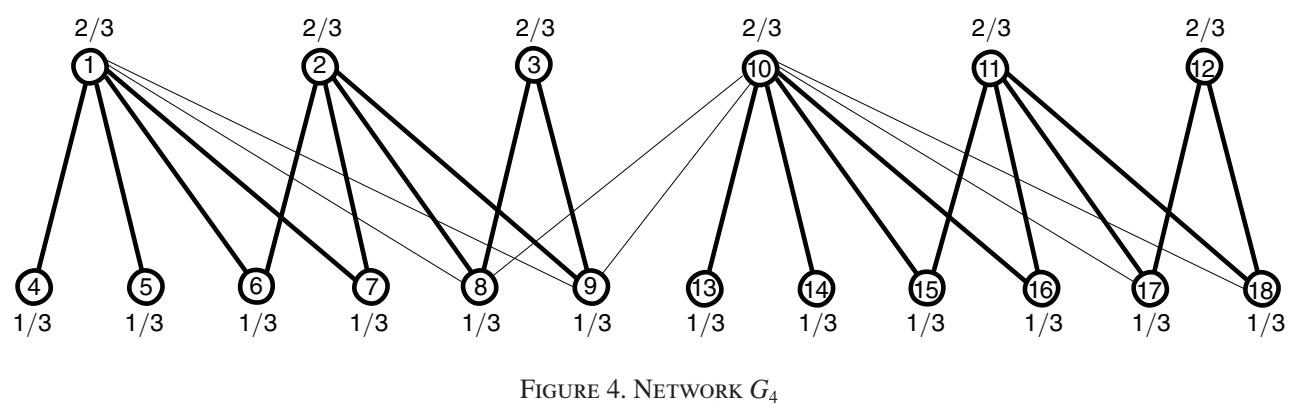

Simple calculus shows that for all $\delta>\underline{\delta}:=6 / 251(45-\sqrt{17}) \approx 0.977$, the following is true for all $i j \in G_{3}: \delta\left(v_{i}^{\delta, G_{3}^{*}}+v_{j}^{\delta, G_{3}^{*}}\right) \leq 1 \Leftrightarrow i j \in G_{3}^{*}$. Hence $v^{\delta, G_{3}^{*}}$ is a fixed point of $f^{\delta}$ and $G_{3}^{* \delta}=G_{3}^{*}$. Therefore, $G_{3}^{*}$ is the limit equilibrium agreement network. For $\delta<\underline{\delta}$, the equilibrium agreement network is the entire $G_{3}$, while for $\delta>\underline{\delta}$ it is $G_{3}^{*}$. The set of equilibria admits a characterization similar to that for the network $G_{2}$ in Example 1.

An example where the players in $M_{s} \cup L_{s}$ induce a connected subnetwork in $G$, but a disconnected subnetwork in $G^{*}$, is provided by the game on the network $G_{4}$ from Figure 4, with the uniform matching technology. 즈 $G_{4}$ essentially consists of two copies of $G_{3}$, with two additional links, $(8,10)$ and $(9,10)$. The limit equilibrium agreement network $G_{4}^{*}$ excludes the links $(1,8),(1,9),(8,10),(9,10),(10,17)$, $(10,18)$ (by a logic analogous to the one suggesting that $G_{3}^{*}$ excludes the links $(1,8)$ and $(1,9))$. 즈

REMARK 5: The results are not sensitive to the assumption that only one pair of players is matched to bargain every period. Consider the following more general matching technology. Suppose that every period nature matches a set (possibly varying in cardinality) of disjoint pairs of linked players. All pairs matched in a given period bargain simultaneously. We assume that the distribution over matchings is stationary and that each link is selected with positive probability. The preliminary results on essential equilibrium uniqueness and convergence as players become patient extend to this setting. Moreover, the limit equilibrium payoffs in the bargaining game with the general matching technology are identical to those from the benchmark model. Relatedly, Section VIII discusses a model with a continuum of players at each node in which bargaining proceeds simultaneously across multiple links.

\section{Equitable Networks}

We call a network equitable if the limit equilibrium payoffs of all players are identical. By Proposition 2, the common limit payoffs must be equal to $1 / 2$. Hence, a network is equitable if a player's position does not affect his bargaining power in

\footnotetext{
${ }^{22}$ See the legend in footnote 2.

${ }^{23}$ The bipartite nature of $G_{3}$ and $G_{4}$ is not critical to the asymptotic results. For instance, the limit equilibrium payoffs and agreement networks for $G_{3}$ and $G_{4}$ remain unchanged if the link $(2,3)$ is added to either network.
} 


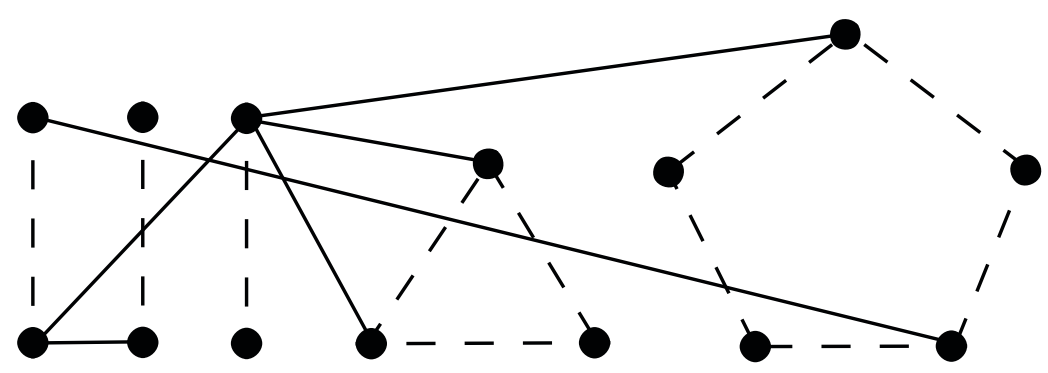

Figure 5. The Dashed Links Constitute an Edge Cover Which Is a Matching AND OdD CyCles DisJoINT UnION

the limit as $\delta$ goes to 1 . Note that the concept of an equitable network is well defined because the limit payoffs in the bargaining game depend on the network structure, but not on the matching technology. We are interested in characterizing the class of equitable networks. By Theorem 4, a network $G$ is equitable if and only if $r_{1} \geq 1$, so that the algorithm $\mathcal{A}(G)$ stops at the first step. Intuitively, this means that no oligopoly emerges in equilibrium. Thus $G$ is equitable if and only if $\left|L^{G}(M)\right| \geq|M|$ for every $G$-independent set $M$. Networks satisfying the latter property have been studied in graph theory. The following definitions are useful.

A graph is an odd cycle if its vertex set has odd cardinality and can be relabeled $1,2, \ldots, k$ such that the set of edges is $\{(1,2),(2,3), \ldots,(k-1, k),(k, 1)\}$. A graph is a matching if its vertex set has even cardinality and can be relabeled $1,2, \ldots, k$ such that the set of edges is $\{(1,2),(3,4), \ldots,(k-1, k)\}$. A graph is a matching and odd cycles disjoint union if it is the union of a matching and a number of odd cycles that are pairwise vertex-disjoint. An edge cover of a graph is a subset of its edges such that every vertex of the graph belongs to at least one edge in the set. A graph is regular if each vertex has the same number of edges.

A graph $H$ is quasi-regularizable (Claude Berge 1981) if there exists $d>0$ and nonnegative integer weights $\omega_{i j}$ associated with each edge $i j \in H$ such that the sum of the weights of the edges incident to any vertex $i$ is $d$,

$$
\sum_{\{j \mid i j \in H\}} \omega_{i j}=d
$$

Examples of quasi-regularizable graphs include regular graphs (set all the weights equal to 1) and matching and odd cycles disjoint unions (set the weights of the edges along the odd cycles equal to 1 and in the matching to 2). If a network is quasi-regularizable then so is any network for which it constitutes an edge cover. Berge (1981) shows that $G$ satisfies $\left|L^{G}(M)\right| \geq|M|$ for every $G$-independent set $M$ if and only if $G$ is quasi-regularizable; another equivalent condition is that $G$ has an edge cover which forms a matching and odd cycles disjoint union. Figure 5 depicts a network that can be covered by a matching and odd cycles disjoint union. Berge's alternative characterizations, along with the discussion opening the section, establish the following result. 


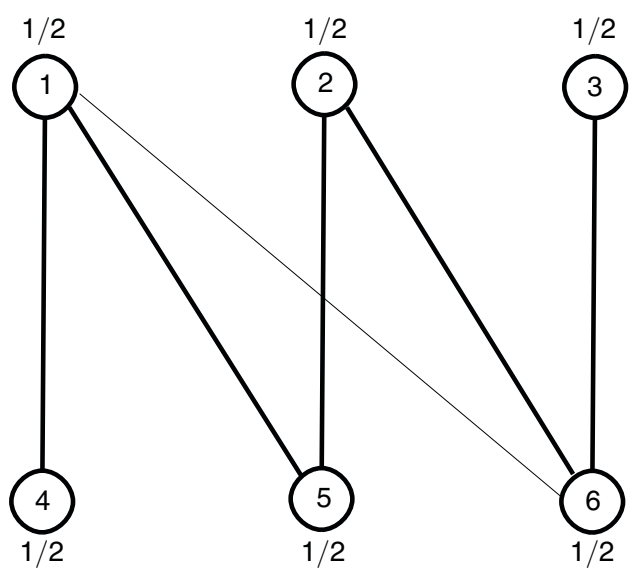

Figure 6. NeTWORK $G_{5}$

THEOREM 5: The following three conditions are equivalent:

(i) $G$ is equitable

(ii) G is quasi-regularizable

(iii) $G$ has an edge cover formed by the disjoint union of a matching and odd cycles.

One important corollary is that regular networks, as well as networks that can be edge covered by regular networks, are equitable.

Example 3: Consider the bargaining game on the six-player network $G_{5}$ drawn in Figure 6, with the uniform matching technology. ${ }^{24}$ By Theorem $5, G_{5}$ is equitable since it has the edge cover $\{(1,4),(2,5),(3,6)\}$, which is a matching. ${ }^{25}$ As the introduction hinted, limit equilibrium payoffs depend on the network structure in a more complex fashion than simply by way of the relative number of bargaining partners. In $G_{5}$ players may have 1,2, or 3 links, but they all receive limit equilibrium payoffs of $1 / 2$. Therefore, while regular networks are equitable, regularity is far from being a necessary condition for equitability. Quasi-regularizability is a necessary and sufficient condition.

Example 4: Suppose that $G$ is the complete network, i.e., $i j \in G, \forall i \neq j \in N$, and that $p_{i j}=\pi_{i} \pi_{j}, \forall i j \in G$, for some vector $\pi$ describing relative matching frequencies $\left(\pi_{i}>0, \forall i \in N\right)$. In this setting, Theorem 5 implies that the limit equilibrium payoff of each player is $1 / 2$, independently of $\pi$. While players with higher matching frequencies obtain larger equilibrium payoffs for any $\delta<1$, all equilibrium payoffs

\footnotetext{
${ }^{24}$ See the legend in footnote 2.

${ }^{25}$ By methods similar to those of Example 2, we can show that the limit equilibrium agreement network excludes the link $(1,6)$.
} 
converge to $1 / 2$ as $\delta$ goes to 1 . The intuition is that patient players can postpone agreement until matched to bargain with players who have low matching frequency (each match occurs with positive probability every period, so every player is eventually matched to a weak partner).

\section{Buyer and Seller Networks}

Suppose that $N=B \cup S$, where $B$ and $S$ denote the sets of buyers and sellers, respectively. Each seller owns one unit of a homogeneous indivisible good. Each buyer demands one unit of the good. The utilities of buyers and sellers for the good are normalized to 1 and 0 , respectively. In a buyer-seller network, links connect buyers to sellers, i.e., for every link $i j, i \in B \Leftrightarrow j \in S$. Fix a buyer-seller network $G$. Only buyer-seller pairs connected in $G$ can engage in exchange. With this interpretation, pairs of agents connected in $G$ can generate a unit surplus, as in the benchmark model. Buyers and sellers have a common discount factor $\delta$ and play the bargaining game $\Gamma^{\delta}$ on the network $G$. Since buyer-seller networks form a special class of networks, all previous results apply, and some refinements are possible.

The bipartite nature of buyer-seller networks permits a more straightforward description of the bounds and of the accompanying procedure for computing limit equilibrium payoffs. The restatements of Theorems 3 and 4 specialized to buyerseller networks are based on the following observations. Fix a buyer-seller network $H$ and a subset of players $M=B^{\prime} \cup S^{\prime}\left(B^{\prime} \subset B, S^{\prime} \subset S\right)$. Then $L^{H}(M)=L^{H}\left(B^{\prime}\right) \cup L^{H}\left(S^{\prime}\right)$ with $L^{H}\left(B^{\prime}\right) \subset S$ and $L^{H}\left(S^{\prime}\right) \subset B$. The set $M$ is $H$-independent if there exists no $H$-link between buyers in $B^{\prime}$ and sellers in $S^{\prime}$, or $L^{H}\left(B^{\prime}\right) \cap S^{\prime}=\varnothing\left(\right.$ or $\left.L^{H}\left(S^{\prime}\right) \cap B^{\prime}=\varnothing\right)$. Also, $B^{\prime}$ and $S^{\prime}$ are $H$-independent.

THEOREM $3^{B S}$ : For every nonempty set of buyers $M$, the following bounds on limit equilibrium payoffs hold:

$$
\begin{aligned}
\min _{i \in M} v_{i}^{*} & \leq \frac{\left|L^{G^{*}}(M)\right|}{|M|+\left|L^{G^{*}}(M)\right|} \\
\max _{j \in L^{G^{*}}(M)} v_{j}^{*} & \geq \frac{|M|}{|M|+\left|L^{G^{*}}(M)\right|} .
\end{aligned}
$$

If we restrict attention to sets of buyers $M$ that minimize $\left|L^{H}(M)\right| /|M|$, Lemma 3 can be extended to show that the set of such minimizers is closed with respect to unions-without the assumption that the attained minimum is strictly less than $1 \longdiv { 2 6 }$

LEMMA $3^{B S}$ : Let $H$ be a buyer-seller network. Suppose that

$$
M^{\prime}, M^{\prime \prime} \in \underset{M \subset B, M \neq \varnothing}{\operatorname{argmin}} \frac{\left|L^{H}(M)\right|}{|M|} .
$$

\footnotetext{
${ }^{26}$ The only addition to the proof of Lemma 3 necessary to adapt it to Lemma $3^{B S}$ is that $A_{4}=A_{5}=\varnothing$ for $M^{\prime}, M^{\prime \prime} \subset B$. Then the weak inequality $(21),(r-1)\left(a_{4}+a_{5}\right) \geq 0$, holds with equality.
} 
Then

$$
M^{\prime} \cup M^{\prime \prime} \in \underset{M \subset B, M \neq \varnothing}{\arg \min } \frac{\left|L^{H}(M)\right|}{|M|} .
$$

In view of Theorem $3^{B S}$ and Lemma $3^{B S}$, the algorithm for computing limit equilibrium payoffs does not have to treat $r_{s} \geq 1$ as a stopping condition at step $s$ if we focus on sets of buyers $M$ that minimize $\left|L^{G_{s}}(M)\right| /|M|$. While the algorithm $\mathcal{A}(G)$ is effective for buyer-seller networks, the adapted version $\mathcal{A}^{B S}(G)$ offers a simplified procedure.

DEFINITION 2: (Algorithm $\left.\mathcal{A}^{B S}(G)=\left(r_{s}, x_{s}, B_{s}, S_{s}, N_{s}, G_{s}\right)_{s=1,2, \ldots, s}\right)$ Define the sequence $\left(r_{s}, x_{s}, B_{s}, S_{s}, N_{s}, G_{s}\right)_{s}$ recursively as follows. Set $N_{1}=N$ and $G_{1}=G$. For $s \geq 1$, let

$$
r_{s}=\min _{M \subset N_{S} \cap B, M \neq \varnothing} \frac{\left|L^{G_{s}}(M)\right|}{|M|} .
$$

Set $x_{s}=r_{s} /\left(1+r_{s}\right)$. Let $B_{s}$ be the union of all minimizers $M$ in (7). 그 Denote $S_{s}=$ $L^{G_{s}}\left(B_{s}\right)$. If $N_{s}=B_{s} \cup S_{s}$ then stop. Otherwise, set $N_{s+1}=N_{s} \backslash\left(B_{s} \cup S_{s}\right)$, and let $G_{s+1}$ be the subnetwork of $G$ induced by the players in $N_{s+1}$. Denote by $\bar{s}$ the finite step at which the algorithm stops.

PROPOSITION $3^{B S}$ : The sequences $\left(r_{s}\right)_{s}$ and $\left(x_{s}\right)_{s}$ defined by $\mathcal{A}^{B S}(G)$ are strictly increasing.

Note that the sets $B_{1}, S_{1}, \ldots, B_{\bar{s}}, S_{\bar{s}}$ partition $N$.

THEOREM $4^{B S}$ : Let $\left(r_{s}, x_{s}, B_{s}, S_{s}, N_{s}, G_{s}\right)_{s=1,2, \ldots, \bar{s}}$ be the outcome of the algorithm $\mathcal{A}^{B S}(G)$ for the buyer-seller network $G$. The limit equilibrium payoffs for $\Gamma^{\delta}$ as $\delta \rightarrow 1$ are given by

$$
\begin{aligned}
& v_{i}^{*}=x_{s}, \forall i \in B_{s}, \forall s \leq \bar{s} \\
& v_{j}^{*}=1-x_{s}, \forall j \in S_{s}, \forall s \leq \bar{s} .
\end{aligned}
$$

Similarly to the study of equitable networks for the general model, we interest ourselves with characterizing the class of nondiscriminatory networks in the bipartite case. A buyer-seller network is nondiscriminatory if the limit equilibrium payoffs of all buyers are identical. By Proposition $3^{B S}$ and Theorem $4^{B S}, G$ is nondiscriminatory if and only if the algorithm $\mathcal{A}^{B S}(G)$ stops at the first step; hence, $B_{1}=B, S_{1}=S, r_{1}=|S| /|B|$. Let $\beta$ denote the buyer-seller ratio, $\beta=|B| /|S|$. In a nondiscriminatory network, the common limit payoffs of all sellers - and the limit equilibrium price-are $\beta /(\beta+1)$.

\footnotetext{
${ }^{27} \mathrm{By}$ Lemma $3^{B S}, B_{s}$ is also a minimizer in (7).
} 
Suppose that $\beta$ is an integer. A $\beta$-buyer-seller cluster is a network formed by a seller connected to $\beta$ buyers. A $\beta$-buyer-seller cluster disjoint union is a network arising from the union of vertex-disjoint $\beta$-buyer-seller clusters. Set $B=\{4,5,6,7,8,9\}$ and $S=\{1,2,3\}$ for the network $G_{3}$ from Example 2 . The buyer-seller ratio is 2 , and the network has an edge cover formed by the disjoint union of three 2-buyer-seller clusters, $\{(1,4),(1,5)\} \cup\{(2,6),(2,7)\} \cup\{(3,8),(3,9)\}$. The network $G_{3}$ is nondiscriminatory, with a limit equilibrium price of $2 / 3$. The intuition is that each seller enjoys a distinct 2-buyer base, so there is no differentiation in bargaining power across patient sellers. This observation can be generalized. The proof is in the Appendix.

THEOREM 6: Suppose that the buyer-seller ratio $\beta$ is an integer. A buyer-seller network is nondiscriminatory if and only if it has an edge cover which is a $\beta$-buyerseller cluster disjoint union.

COROLLARY 2: A buyer-seller network is equitable if and only if it has a perfect matching. 28

REMARK 6: Corollary 2 also follows from Theorem 5 since bipartite networks contain no odd cycles, so any matching and odd cycle disjoint union that covers such a network must be a perfect matching.

REMARK 7: Analogous results obtain if we interchange the roles of buyers and sellers in Theorems $4^{B S}$ and 6.

REMARK 8: Theorem $4^{B S}$ extends to a setting with the following bargaining protocol. Suppose that in every match the seller is $q \geq 0$ times more likely than the buyer to be the proposer. Note that the benchmark model presumes $q=1$. Consider the sequence $\left(r_{s}, x_{s}, B_{s}, S_{s}, N_{s}, G_{s}\right)_{s}$ generated by the algorithm $\mathcal{A}^{B S}(G)$, with the variable $x_{s}$ redefined as $r_{s} /\left(q+r_{s}\right)$ for all $s$ and all other variables left intact. The sequence delivers the limit equilibrium payoffs for the new game as detailed in Theorem $4^{B S}$.

\section{Steady States with a Continuum of Players}

The results translate to an alternative specification of the model in the spirit of Gale (1987). We fix $G$ and $\delta$ throughout this section. Suppose there is a continuum of players in the market every period. We refer to the players at node $i \in G$ as players of type $i$ or simply players $i$. A measure $\mu_{i}$ of players of type $i$ participate in the game every period. The vector $\mu=\left(\mu_{i}\right)_{i \in G}$ defines a (static) market. The matching technology is such that, for each link $i j \in G$, a measure $\beta_{i j}(\mu)$ of players $i$ are matched to bargain with one of the players $j$. It is assumed that $\beta_{i j}(\mu)=\beta_{j i}(\mu)>0$ and $\mu_{i}>\sum_{\{j \mid i j \in G\}} \beta_{i j}(\mu)$. All players of the same type are treated symmetrically, so the probability that a player $i$ meets some player $j$ is $\beta_{i j}(\mu) / \mu_{i}$. For each matched pair, bargaining proceeds through random selection

\footnotetext{
${ }^{28}$ A perfect matching of a network is a matching that constitutes an edge cover.
} 
of the proposer. To maintain the bilateral feature of the model, we require that no player be involved in more than one match at the same time.

For a concrete example of a matching technology, assume that every player $i$ meets another player with a fixed probability $p$, and the conditional probability of $i$ meeting a type $j$ is proportional to the measure of players $j$ in the market. In our network setting, a meeting between $i$ and $j$ results in a feasible match only if $i j \in G$. The corresponding matching technology is described by $\beta_{i j}(\mu)=p \mu_{i} \mu_{j} / \sum_{k \in G} \mu_{k}$ if $i j \in G$ and $\beta_{i j}(\mu)=0$ otherwise (cf. Gale 1987).

To preserve the stationarity of the game, we have to assume that the set of players of each type who reach agreements is immediately replaced by an equal measure of new players of the same type. ${ }^{29}$ Hence the distribution of players in the network at the beginning of each period is given by $\mu$. The steady state analysis of the previous sections extends here without difficulty.

In particular, the game $\Gamma(\mu)$, corresponding to the stationary market $\mu$, yields a unique equilibrium payoff $v_{i}(\mu)$ to all players present at node $i$ at the beginning of any round. The analog of Theorem 1 is that for every $\mu \in(0, \infty)^{n}, v(\mu)$ is the unique fixed point of the contraction $f:[0,1]^{n} \rightarrow[0,1]^{n}$ defined by

$$
f_{i}(v)=\left(1-\sum_{\{j \mid i j \in G\}} \frac{\beta_{i j}(\mu)}{2 \mu_{i}}\right) \delta v_{i}+\sum_{\{j \mid i j \in G\}} \frac{\beta_{i j}(\mu)}{2 \mu_{i}} \max \left(1-\delta v_{j}, \delta v_{i}\right) .
$$

Furthermore, the results of Theorems 3 and 4 regarding limit equilibrium payoffs as $\delta \rightarrow 1$ carry over to the current setting if the shortage ratio of a mutually estranged set $M$ with partner set $L$ is evaluated using the formula

$$
\frac{\sum_{j \in L} \mu_{j}}{\sum_{i \in M} \mu_{i}}
$$

While we are able to provide a detailed characterization of the equilibrium payoffs and agreements in any stationary market, we have yet to address the issue of determining a steady state in a setting where the set of potential market entrants is exogenous. Specifically, suppose that every period a measure $\lambda_{i}>0$ of players of type $i$ may choose to enter the game. Manea (2010) points out that even without any equilibrium restriction on the set of agreements, regardless of the matching technology $\beta$, it may be that no feasible mass of pairwise departures from the market perfectly balances the inflows $\lambda$. This motivates the introduction of small entry costs to restrict the equilibrium inflows and stabilize the market.

In addition to $G$ and $\delta$, the other primitives of the model are the inflows $\lambda=\left(\lambda_{i}>0\right)_{i \in G}$ and the (population specific) entry costs $c=\left(c_{i}\right)_{i \in G}$. An economy is defined by the quadruple $(G, \delta, \lambda, c)$. When does a distribution $\mu$ of players across $G$ constitute a steady state market for the economy $(G, \delta, \lambda, c)$ ? It must be that every player of type $i$ enters the game $\Gamma(\mu)$ if $v_{i}(\mu)>c_{i}$ and does not if $v_{i}(\mu)<c_{i}$. Hence the measure of new entrants in population $i$ is $\lambda_{i}$ if $v_{i}(\mu)>c_{i}, 0$ if $v_{i}(\mu)<c_{i}$, and any number in the interval $\left[0, \lambda_{i}\right]$ if $v_{i}(\mu)=c_{i}$. The measure of players $i$ trading with

\footnotetext{
${ }^{29}$ Manea (2010) discusses technical restrictions on strategies that guarantee the measurability of the set of players who trade.
} 
players $j$ in any period is $\beta_{i j}(\mu), 0$, or any number in $\left[0, \beta_{i j}(\mu)\right]$ depending on whether the sum of continuation payoffs $\delta\left(v_{i}(\mu)+v_{j}(\mu)\right)$ is less than, greater than, or equal to the unit surplus, respectively. The steady state condition requires that the measure of players $i$ entering the game in any period must be identical to the total measure of players $i$ who reach agreement that period. A formal definition of steady states can be found in Manea (2010).

The latter paper provides an example in which steady states do not exist for a wide range of vectors of small entry costs. The next result, which we prove in the general setting of that paper, asserts that if the matching technology is continuous then for any given inflows $\lambda$ there exist small entry costs $c$ such that the economy $(G, \delta, \lambda, c)$ admits a steady state.

THEOREM 7: Fix the network $G$, the discount factor $\delta$, a matching technology $\beta$ with $\beta_{i j}$ continuous on $(0, \infty)^{n}$ for all $i j \in G$, and constant inflows $\lambda$. Then for every $k>0$, there exist entry costs $\mathrm{c}$ with $c_{i}<k$ for all $i \in G$ such that the economy $(G, \delta, \lambda, c)$ has a steady state $\mu \in(0, \infty)^{n}$.

\section{Related Literature}

Rubinstein and Wolinsky (1985) provided a major contribution to the analysis of decentralized trade in stationary markets. ${ }^{-3}$ Apart from some insignificant differences, their model is embedded in our setting as the special case of a buyer-seller network in which every buyer is connected to every seller. Rubinstein and Wolinsky found that, as players become patient, the surplus is divided between buyers and sellers according to the ratio of sellers to buyers. Our results develop the idea that, in stationary environments with complex patterns of transactions, the shortage ratio of every mutually estranged set describes the collective bargaining power of its members.

In Dilip Abreu and Manea (2009a, b), we drop the stationarity assumption and analyze the situation in which players who reach agreements are removed from the network without replacement. The matching technology and the bargaining protocol are identical to those of the present paper. In that setting, there are networks where no Markov perfect equilibrium is asymptotically efficient as players become patient. An important result is that for every network asymptotic efficiency is attainable in a subgame perfect equilibrium. We also investigate properties of Markov perfect equilibria.

The two settings differ in strategic complexity. In the present model bargaining opportunities are stationary over time. A player's decisions consist solely in determining who his most favorable bargaining partners are. In effect, each player solves a search problem with prizes endogenously and simultaneously determined by the network structure. In the setting without replacement, a player's decisions additionally entail anticipating that passing up bargaining opportunities may lead to agreements involving other players which undermine or enhance his position in the network in future bargaining encounters. Technically, this means that we need to compute equilibrium payoffs for every subnetwork that may arise following a series of agreements. Clearly, the selection between the two models depends on the

\footnotetext{
${ }^{30}$ Important follow-up studies of bargaining in markets include Gale (1987), Binmore and Herrero (1988a), and Rubinstein and Wolinsky (1990).
} 
environment under investigation. In some markets trading opportunities are stationary, while in others trade volume declines over time.

Manea (2010) considers general models of bargaining in markets with multiple populations and exogenous-possibly nonstationary-inflows into each population. The surplus generated from trade is heterogenous across population pairs. We explore several properties of equilibria, including existence, multiplicity, and efficiency. We also provide an analysis of steady state economies.

Arnold Polanski (2007) introduced a model related to Abreu and Manea (2009a, b). The difference lies in the matching technology, as Polanski assumes that a maximum number of pairs of linked players are selected to bargain every period. Margarida Corominas-Bosch (2004) considered a game in which buyers and sellers alternate in making public offers. Each player can accept offers from any of the proposers connected to him. When there are multiple possibilities to clear the market, the maximum number of transactions takes place. The efficient matching technologies of Corominas-Bosch and Polanski are fundamentally centralized. The market forces that would organize the matchings to maximize total surplus are not explicitly modeled by way of self-interested strategic behavior.

Centralized trading mechanisms may be employed to implement efficient matching outcomes. In the framework of Rachel E. Kranton and Deborah F. Minehart (2001), buyers have heterogeneous valuations, and sellers are nonstrategic. Prices are determined by the simultaneous ascending-bid auction mechanism introduced by Vincent P. Crawford and Elsie Marie Knoer (1981). The unique equilibrium in weakly undominated strategies leads to an efficient allocation of the goods.

\section{Conclusion}

Networks are important in many economic and social interactions. In our setting networks represent patterns of trading opportunities. The network structure affects the set of feasible agreements, the division of surplus, and the relative bargaining strengths. Previous studies of surplus division in networks mainly considered ad hoc allocation rules or cooperative solution concepts. Some papers discussed in the literature review analyze noncooperative division of surplus under centralized mechanisms. Yet only a few papers have explored the strategic issues that arise in bargaining in networks with random matching. The models of noncooperative decentralized bargaining in networks of the present paper and Abreu and Manea (2009a, b) constitute initial endeavors in that exploration.

The model introduced here is well behaved in that equilibria are essentially unique and converge as players become patient. The main result of the paper is the characterization of the limit equilibrium payoffs by iterative use of the following findings. Limit payoffs are lowest for the players in the largest mutually estranged set that minimizes the shortage ratio, and highest for the corresponding partners. In equilibrium, for high discount factors, the partners act as an oligopoly for the estranged players. In the limit, surplus within the induced oligopoly subnetwork is divided according to the shortage ratio. The ideas of mutually estranged sets and minimal shortage ratios, along with induced oligopoly subnetworks, provide insights into the relative strengths of the positions in a network. The limit equilibrium payoffs deliver an index of bargaining power in stationary networks. 


\section{APPENDIX}

LEMMA 5: For all $w_{1}, w_{2}, w_{3}, w_{4} \in \mathbb{R}$,

$$
\left|\max \left(w_{1}, w_{2}\right)-\max \left(w_{3}, w_{4}\right)\right| \leq \max \left(\left|w_{1}-w_{3}\right|,\left|w_{2}-w_{4}\right|\right)
$$

\section{PROOF OF LEMMA 5:}

Suppose $w_{1}=\max \left(w_{1}, w_{2}, w_{3}, w_{4}\right)$. Then

$$
\begin{aligned}
\left|\max \left(w_{1}, w_{2}\right)-\max \left(w_{3}, w_{4}\right)\right| & =w_{1}-\max \left(w_{3}, w_{4}\right) \\
& \leq w_{1}-w_{3} \leq \max \left(\left|w_{1}-w_{3}\right|,\left|w_{2}-w_{4}\right|\right) .
\end{aligned}
$$

The proof is similar for the cases when $w_{2}, w_{3}$, or $w_{4}$ is equal to $\max \left(w_{1}, w_{2}, w_{3}, w_{4}\right)$.

LEMMA 6: The function $f^{\delta}:[0,1]^{n} \rightarrow[0,1]^{n}$ defined by (2) is a contraction with respect to the sup norm on $\mathbb{R}^{n}$.

\section{PROOF OF LEMMA 6:}

Recall that the sup norm $\|\cdot\|$ on $\mathbb{R}^{n}$ is defined by $\|z\|=\max _{i=\overline{1, n}}\left|z_{i}\right|$. We argue that

$$
\left\|f^{\delta}(v)-f^{\delta}(u)\right\| \leq \delta\|v-u\|, \forall v, u \in[0,1]^{n} .
$$

We need to prove that for each $i,\left|f_{i}^{\delta}(v)-f_{i}^{\delta}(u)\right| \leq \delta\|v-u\|$. By $f^{\delta}$, s definition,

$$
\begin{aligned}
\left|f_{i}^{\delta}(v)-f_{i}^{\delta}(u)\right| \leq & \left(1-\sum_{\{j \mid i j \in G\}} \frac{p_{i j}}{2}\right) \delta\left|v_{i}-u_{i}\right| \\
& +\sum_{\{j \mid i j \in G\}} \frac{p_{i j}}{2}\left|\max \left(1-\delta v_{j}, \delta v_{i}\right)-\max \left(1-\delta u_{j}, \delta u_{i}\right)\right| \\
\leq & \left(1-\sum_{\{j \mid i j \in G\}} \frac{p_{i j}}{2}\right) \delta\left|v_{i}-u_{i}\right| \\
& +\sum_{\{j \mid i j \in G\}} \frac{p_{i j}}{2} \max \left(\left|1-\delta v_{j}-\left(1-\delta u_{j}\right)\right|,\left|\delta v_{i}-\delta u_{i}\right|\right) \\
= & \left(1-\sum_{\{j \mid i j \in G\}} \frac{p_{i j}}{2}\right) \delta\left|v_{i}-u_{i}\right| \\
& +\sum_{\{j \mid i j \in G\}} \frac{p_{i j}}{2} \delta \max \left(\left|v_{j}-u_{j}\right|,\left|v_{i}-u_{i}\right|\right) \\
\leq & \left(1-\sum_{\{j \mid i j \in G\}} \frac{p_{i j}}{2}\right) \delta\|v-u\|+\sum_{\{j \mid i j \in G\}} \frac{p_{i j}}{2} \delta\|v-u\| \\
= & \delta \| v-u_{\|}, v_{1}
\end{aligned}
$$


where the second inequality follows from Lemma 5, and the others from algebraic manipulation and the definition of the sup norm.

\section{PROOF OF PROPOSITION 1:}

By definition, $i j \in G^{* \delta} \Leftrightarrow i j \in G \& \max \left(1-\delta v_{j}^{* \delta}, \delta v_{i}^{* \delta}\right)=1-\delta v_{j}^{* \delta}$. Since $v^{* \delta}$ is a fixed point of $f^{\delta}, v^{* \delta}$ solves the $n \times n$ system of linear equations

$$
v_{i}=\left(1-\sum_{\left\{j \mid i j \in G^{* \delta}\right\}} \frac{p_{i j}}{2}\right) \delta v_{i}+\sum_{\left\{j \mid i j \in G^{* \delta}\right\}} \frac{p_{i j}}{2}\left(1-\delta v_{j}\right), \forall i=\overline{1, n} .
$$

For every $\delta \in(0,1)$ and every nonempty subnetwork $H$ of $G$, consider more generally the $n \times n$ linear system (3). We argue below that the system has a unique solution $v^{\delta, H}$, and the solution belongs to $[0,1]^{n}$. In particular, the system $(3)$ is nonsingular and $v^{* \delta}=v^{\delta, G^{* \delta}}$.

The simplest path to show uniqueness of the solution to (3) is analytical rather than linear algebraic, by proving that the function $h^{\delta, H}: \mathbb{R}^{n} \rightarrow \mathbb{R}^{n}$ defined by

$$
h_{i}^{\delta, H}(v)=\left(1-\sum_{\{j \mid i j \in H\}} \frac{p_{i j}}{2}\right) \delta v_{i}+\sum_{\{j \mid i j \in H\}} \frac{p_{i j}}{2}\left(1-\delta v_{j}\right), \forall i=\overline{1, n}
$$

is a contraction with respect to the sup norm on $\mathbb{R}^{n}$. The proof is omitted as it is similar (but simpler, since it does not involve Lemma 5) to that of Lemma 6. The unique fixed point belongs to $[0,1]^{n}$ since $h^{\delta, H}\left([0,1]^{n}\right) \subset[0,1]^{n}$.

All entries in the augmented matrix of the linear system (3) are linear functions of $\delta$. Then for each $i \in N$ the solution $v_{i}^{\delta, H}$ is given by Cramer's rule, as the ratio of two determinants that are polynomials in $\delta$ of degree at most $n$,

$$
v_{i}^{\delta, H}=P_{i}^{H}(\delta) / Q_{i}^{H}(\delta)
$$

Note that $Q_{i}^{H}(\delta) \neq 0$ for all $\delta \in(0,1)$ and all nonempty subnetworks $H$ of $G$ as the corresponding system $(3)$ is nonsingular.

Let $\bar{\Delta}$ be the set of $\delta$ for which there exist $i, j, H$ with $\delta\left(v_{i}^{\delta, H}+v_{j}^{\delta, H}\right)=1$. Fix $i, j, H$. The equation $\delta\left(v_{i}^{\delta, H}+v_{j}^{\delta, H}\right)=1$ is equivalent to

$$
1=\delta\left(v_{i}^{\delta, H}+v_{j}^{\delta, H}\right)=\delta\left(P_{i}^{H}(\delta) / Q_{i}^{H}(\delta)+P_{j}^{H}(\delta) / Q_{j}^{H}(\delta)\right) .
$$

If the equation above has an infinite number of solutions $\delta$, it follows that

$$
\delta\left(P_{i}^{H}(\delta) Q_{j}^{H}(\delta)+P_{j}^{H}(\delta) Q_{i}^{H}(\delta)\right)=Q_{i}^{H}(\delta) Q_{j}^{H}(\delta)
$$

is a polynomial identity, i.e., it holds for all $\delta$. In particular, equality needs to hold in the two display equations above for $\delta=1 / 3$, implying that $1 / 3\left(v_{i}^{1 / 3, H}+v_{j}^{1 / 3, H}\right)=1$. Hence $v_{i}^{1 / 3, H}+v_{j}^{1 / 3, H}=3$, which is a contradiction with $v^{1 / 3, H} \in[0,1]^{n}$.

Since for every triple $(i, j, H)$ the equation $\delta\left(v_{i}^{\delta, H}+v_{j}^{\delta, H}\right)=1$ has a finite number of solutions $\delta$, and the number of such triples is finite, it follows that the set $\bar{\Delta}$ is finite. The equality $v^{* \delta}=v^{\delta, G^{* \delta}}$ implies that the set of $\delta$ for which there exist $i, j$ s.t. $\delta\left(v_{i}^{* \delta}+v_{j}^{* \delta}\right)=1$ is included in $\bar{\Delta}$. 


\section{PROOF THEOREM 2:}

To establish the first part, recall that the proof of Proposition 1 shows that the set $\bar{\Delta}$ of $\delta$ for which there exist a link $i j \in G$ and a subnetwork $H$ of $G$ such that $\delta\left(v_{i}^{\delta, H}+v_{j}^{\delta, H}\right)=1$ is finite. Fix $\delta_{0}>\max \bar{\Delta}=: \underline{\delta}$. Let $G^{*}=G^{* \delta_{0}}$. We show that $G^{* \delta}=G^{*}$ for all $\delta>\underline{\delta}$.

Fix $i j \in G$. The function $1-\delta\left(v_{i}^{\delta, G^{*}}+v_{j}^{\delta, G^{*}}\right)$ is continuous in $\delta$ for $\delta \in(0,1)$ as it is a rational function (ratio of two polynomials) by (8), and it has no roots $\delta$ outside $\bar{\Delta}$. Then the $\operatorname{sign} \varepsilon_{i j}^{\delta}$ of $1-\delta\left(v_{i}^{\delta, G^{*}}+v_{j}^{\delta, G^{*}}\right)$ is strict and constant for all $\delta>$ $\underline{\delta}$. In particular, $\varepsilon_{i j}^{\delta}=\varepsilon_{i j}^{\delta_{0}}$ for $\delta>\underline{\delta}$.

Since $\delta_{0}>\underline{\delta}$ and $G^{*}=G^{* \delta_{0}}$, the following conditions hold (1) $\varepsilon_{i j}^{\delta_{0}}=1 \Leftrightarrow i j \in G^{*}$ and (2) $\varepsilon_{i j}^{\delta_{0}}=-1 \Leftrightarrow i j \notin G^{*}$. For all $\delta>\underline{\delta}$ we have $\varepsilon_{i j}^{\delta}=\varepsilon_{i j}^{\delta_{0}}$; hence, the following conditions must also be true: (1) $\varepsilon_{i j}^{\delta}=1 \Leftrightarrow i j \in G^{*}$ and (2) $\varepsilon_{i j}^{\delta}=-1 \Leftrightarrow i j \notin G^{*}$. For $\delta>\underline{\delta}$, it follows that $v^{\delta, G^{*}}$ is a fixed point of $f^{\delta}$ (defined by $\left.(2)\right)$, hence $v^{* \delta}=v^{\delta, G^{*}}$ and $G^{*} \delta=G^{*}$.

To establish the second part, fix $i \in N$. From the first part, $v_{i}^{* \delta}=P_{i}^{G^{*}}(\delta) / Q_{i}^{G^{*}}(\delta)$ for $\delta>\underline{\delta}$. Rewrite $P_{i}^{G^{*}} / Q_{i}^{G^{*}}=\bar{P}_{i}^{G^{*}} / \bar{Q}_{i}^{G^{*}}$, with $\bar{P}_{i}^{G^{*}}$ and $\bar{Q}_{i}^{G^{*}}$ relatively prime polynomials. Since $v_{i}^{* \delta} \in[0,1]$ for all $\delta \in(\underline{\delta}, 1)$, it must be that $\bar{Q}_{i}^{G^{*}}(1) \neq 0$. For, if $\bar{Q}_{i}^{G^{*}}(1)=0$ then $\bar{P}_{i}^{G^{*}}(1) \neq 0$ (as $\bar{P}_{i}^{G^{*}}$ and $\bar{Q}_{i}^{G^{*}}$ are relatively prime) and $\left|\bar{P}_{i}^{G^{*}}(\delta) / \bar{Q}_{i}^{G^{*}}(\delta)\right|$ diverges to infinity as $\delta \rightarrow 1$. Consequently, $\bar{P}_{i}^{G^{*}}(\delta) / \bar{Q}_{i}^{G^{*}}(\delta)$ converges to $\bar{P}_{i}^{G^{*}}(1) / \bar{Q}_{i}^{G^{*}}(1)$ as $\delta$ tends to 1 . Therefore, $v_{i}^{* \delta}=\bar{P}_{i}^{G^{*}}(\delta) / \bar{Q}_{i}^{G^{*}}(\delta)$ has a finite limit, $v_{i}^{*}:=\bar{P}_{i}^{G^{*}}(1) / \bar{Q}_{i}^{G^{*}}(1)$, at $\delta=1$.

To show that the rate of convergence of $v^{*} \delta$ to $v^{*}$ is $O(1-\delta)$, write

$$
v_{i}^{* \delta}-v_{i}^{*}=\frac{\bar{P}_{i}^{G^{*}}(\delta)}{\bar{Q}_{i}^{G^{*}}(\delta)}-\frac{\bar{P}_{i}^{G^{*}}(1)}{\bar{Q}_{i}^{G^{*}}(1)}=\frac{\bar{Q}_{i}^{G^{*}}(1) \bar{P}_{i}^{G^{*}}(\delta)-\bar{P}_{i}^{G^{*}}(1) \bar{Q}_{i}^{G^{*}}(\delta)}{\bar{Q}_{i}^{G^{*}}(1) \bar{Q}_{i}^{G^{*}}(\delta)} .
$$

The latter rational function has a vanishing numerator and a nonvanishing denominator at $\delta=1$; hence, it can be rewritten as $(1-\delta) R_{i}^{G^{*}}(\delta)$, where $R_{i}^{G^{*}}$ is a rational function with a finite limit at $\delta=1$.

\section{PROOF OF PROPOSITION 2:}

Let $i j \in G$. If $i j \in G \backslash G^{*}$, then for all $\delta>\underline{\delta}$,

$$
\delta\left(v_{i}^{* \delta}+v_{j}^{* \delta}\right)>1 .
$$

If $i j \in G^{*}$, then for all $\delta>\underline{\delta}$,

$$
\begin{aligned}
v_{i}^{* \delta} & =\left(1-\sum_{\left\{k \mid i k \in G^{*}\right\}} \frac{p_{i k}}{2}\right) \delta v_{i}^{* \delta}+\sum_{\left\{k \mid i k \in G^{*}\right\}} \frac{p_{i k}}{2}\left(1-\delta v_{k}^{* \delta}\right) \\
& \geq\left(1-\frac{p_{i j}}{2}\right) \delta v_{i}^{* \delta}+\frac{p_{i j}}{2}\left(1-\delta v_{j}^{* \delta}\right),
\end{aligned}
$$

since $1-\delta v_{k}^{* \delta} \geq \delta v_{i}^{* \delta}$ for all $k \neq j$ such that $i k \in G^{*}$. Taking the limit as $\delta$ goes to 1 in either (9) or (10) we obtain that $v_{i}^{*}+v_{j}^{*} \geq 1$.

\footnotetext{
${ }^{31}$ For all $\delta \in(0,1), Q_{i}^{G^{*}}(\delta) \neq 0$ because the system (3) is nonsingular.
} 
In conclusion, $v_{i}^{*}+v_{j}^{*} \geq 1, \forall i j \in G$. The other claims follow similarly.

\section{PROOF OF LEMMA 1:}

Fix $\delta>\underline{\delta}$. If $i$ had no link in $G^{*}$, then $v_{i}^{* \delta}=0$. By footnote 5, there exists $j$ such that $i j \in G$. Then $\delta\left(v_{i}^{* \delta}+v_{j}^{*}\right)<1$, which means that $i j \in G^{*}$, a contradiction.

\section{PROOF OF THEOREM 3:}

Let $M$ be a mutually estranged set with partner set $L$. Fix $\delta>\underline{\delta}$, with $\underline{\delta}$ specified as in Theorem 2. Then in every equilibrium of $\Gamma^{\delta}$, a pair of players connected in $G$ reach agreement when matched to bargain if and only if they are connected in $G^{*}$.

By Lemma 2, for all $i$ in $M$,

$$
\begin{aligned}
v_{i}^{* \delta} & =\frac{1}{1-\delta} \sum_{\{j \mid i j \in G\}} \frac{p_{i j}}{2} \max \left(1-\delta v_{i}^{* \delta}-\delta v_{j}^{* \delta}, 0\right) \\
& =\frac{1}{1-\delta} \sum_{\{j \mid i j \in G, j \in L\}} \frac{p_{i j}}{2} \max \left(1-\delta v_{i}^{* \delta}-\delta v_{j}^{* \delta}, 0\right),
\end{aligned}
$$

since $i$ has $G^{*}$ links only to players in $L$, so $\max \left(1-\delta v_{i}^{* \delta}-\delta v_{j}^{* \delta}, 0\right)=0$ if $i j \in G$, $j \notin L$.

By Lemma 2, for all $j$ in $L$,

$$
\begin{aligned}
v_{j}^{* \delta} & =\frac{1}{1-\delta} \sum_{\{i \mid i j \in G\}} \frac{p_{i j}}{2} \max \left(1-\delta v_{i}^{* \delta}-\delta v_{j}^{* \delta}, 0\right) \\
& \geq \frac{1}{1-\delta} \sum_{\{i \mid i j \in G, i \in M\}} \frac{p_{i j}}{2} \max \left(1-\delta v_{i}^{* \delta}-\delta v_{j}^{* \delta}, 0\right) .
\end{aligned}
$$

Adding up the equalities (11) across all $i \in M$ and the inequalities (12) across all $j \in L$ we obtain

$$
\begin{aligned}
& \sum_{i \in M} v_{i}^{* \delta}=\frac{1}{1-\delta} \sum_{\{(i, j) \mid i j \in G, i \in M, j \in L\}} \frac{p_{i j}}{2} \max \left(1-\delta v_{i}^{* \delta}-\delta v_{j}^{* \delta}, 0\right) \\
& \sum_{j \in L} v_{j}^{* \delta} \geq \frac{1}{1-\delta} \sum_{\{(i, j) \mid i j \in G, i \in M, j \in L\}} \frac{p_{i j}}{2} \max \left(1-\delta v_{i}^{* \delta}-\delta v_{j}^{* \delta}, 0\right) .
\end{aligned}
$$

Therefore,

$$
\sum_{j \in L} v_{j}^{* \delta} \geq \sum_{i \in M} v_{i}^{* \delta}
$$

which after taking the limit $\delta \rightarrow 1$ becomes

$$
\sum_{j \in L} v_{j}^{*} \geq \sum_{i \in M} v_{i}^{*}
$$


We can manipulate the latter inequality to obtain that

$$
|L| \max _{j \in L} v_{j}^{*} \geq|M| \min _{i \in M} v_{i}^{*}
$$

Player $\underline{i} \in \arg \min _{i \in M} v_{i}^{*}$ is connected in $G^{*}$ to a player $\tilde{j} \in L$ (Lemma 1$)$; hence, by Proposition 2, $v_{i}^{*}+v_{\tilde{j}}^{*}=1$. Thus $\min _{i \in M} v_{i}^{*}=1-v_{\tilde{j}}^{*} \geq 1-\max _{j \in L} v_{j}^{*}$.

Also, any $\bar{j} \in \arg \max _{j \in L} v_{j}^{*}$ is connected in $G^{*}$ to a player $\tilde{i} \in M$, and $v_{\tilde{i}}^{*}+v_{\bar{j}}^{*}=1$ by Proposition 2 . Hence, $\max _{j \in L} v_{j}^{*}=1-v_{i}^{*} \leq 1-\min _{i \in M} v_{i}^{*}$.

We proved that $\min _{i \in M} v_{i}^{*}=1-\max _{j \in L} v_{j}^{*}$. It follows that

$$
|L| \max _{j \in L} v_{j}^{*} \geq|M|\left(1-\max _{j \in L} v_{j}^{*}\right),
$$

which is equivalent to

$$
\max _{j \in L} v_{j}^{*} \geq \frac{|M|}{|M|+|L|}
$$

Moreover,

$$
\min _{i \in M} v_{i}^{*}=1-\max _{j \in L} v_{j}^{*} \leq 1-\frac{|M|}{|M|+|L|}=\frac{|L|}{|M|+|L|} .
$$

\section{PROOF OF LEMMA 3:}

Suppose that $r:=\min _{M \in \mathcal{I}(H)}\left|L^{H}(M)\right| /|M|<1$, and let $M^{\prime}, M^{\prime \prime}$ be two $H$-independent sets achieving the minimum. Decompose the set $M^{\prime}$ as the union of the sets $A_{2}=M^{\prime} \cap M^{\prime \prime}, A_{1}=\left(M^{\prime} \backslash M^{\prime \prime}\right) \backslash L^{H}\left(M^{\prime \prime}\right)$ (the set of players in $M^{\prime} \backslash M^{\prime \prime}$ who do not have any $H$ links to $\left.M^{\prime \prime}\right)$, and $A_{4}=\left(M^{\prime} \backslash M^{\prime \prime}\right) \cap L^{H}\left(M^{\prime \prime}\right)$ (the set of players in $M^{\prime} \backslash M^{\prime \prime}$ who have $H$ links to $\left.M^{\prime \prime}\right)$. Similarly, decompose the set $M^{\prime \prime}$ as the union of $A_{2}, A_{3}=\left(M^{\prime \prime} \backslash M^{\prime}\right) \backslash L^{H}\left(M^{\prime}\right)$, and $A_{5}=\left(M^{\prime \prime} \backslash M^{\prime}\right) \cap L^{H}\left(M^{\prime}\right)$. Let $B_{2}=L^{H}\left(A_{2}\right), B_{1}=$ $L^{H}\left(A_{1}\right) \backslash B_{2}, B_{3}=L^{H}\left(A_{3}\right) \backslash B_{2}$. Denote $\left|A_{i}\right|=a_{i},\left|B_{j}\right|=b_{j}$ for $i=\overline{1,5}, j=\overline{1,3}$.

Since $M^{\prime \prime}$ is $H$-independent, there are no $H$ links between $A_{5}$ and $A_{2}$. Also, there are no $H$ links between $A_{5}$ and $A_{1}$ because $A_{1} \cap L^{H}\left(M^{\prime \prime}\right)=\varnothing$. Then, as $L^{H}\left(M^{\prime}\right) \supset$ $A_{5}$, it must be that $L^{H}\left(A_{4}\right) \supset A_{5}$. Similarly, $L^{H}\left(A_{5}\right) \supset A_{4}$. Therefore, 22

$$
\begin{aligned}
L^{H}\left(A_{1} \cup A_{2} \cup A_{3}\right) & =B_{1} \cup B_{2} \cup B_{3} \\
L^{H}\left(M^{\prime}\right) & =L^{H}\left(A_{1} \cup A_{2} \cup A_{4}\right) \supset B_{1} \cup B_{2} \cup A_{5} \\
L^{H}\left(M^{\prime \prime}\right) & =L^{H}\left(A_{2} \cup A_{3} \cup A_{5}\right) \supset B_{2} \cup B_{3} \cup A_{4} \\
L^{H}\left(A_{2}\right) & =B_{2} .
\end{aligned}
$$

\footnotetext{
${ }^{32}$ The middle two expressions can be strict inclusions as players in $A_{4}\left(A_{5}\right)$ may have $H$ links to players outside $B_{1} \cup B_{2} \cup A_{5}\left(B_{2} \cup B_{3} \cup A_{4}\right)$.
} 
Since there are no $H$ links between $A_{1} \cup A_{2}$ and $M^{\prime \prime}$, it follows that $\left(B_{1} \cup B_{2}\right) \cap$ $A_{5} \subset\left(B_{1} \cup B_{2}\right) \cap M^{\prime \prime}=\varnothing$. Analogously, $\left(B_{2} \cup B_{3}\right) \cap A_{4}=\varnothing$. By definition, $B_{1} \cap$ $B_{2}=\varnothing$ and $B_{2} \cap B_{3}=\varnothing$. It follows that the triples $\left(B_{1}, B_{2}, A_{5}\right)$ and $\left(B_{2}, B_{3}, A_{4}\right)$ consist of pairwise disjoint sets; hence, $\left|B_{1} \cup B_{2} \cup A_{5}\right|=b_{1}+b_{2}+a_{5}$ and $\left|B_{2} \cup B_{3} \cup A_{4}\right|=$ $b_{2}+b_{3}+a_{4}$. The intersection of $B_{1}$ and $B_{3}$ may be nonempty; hence, $\left|B_{1} \cup B_{2} \cup B_{3}\right| \leq$ $b_{1}+b_{2}+b_{3}$.

The definitions of $r, M^{\prime}, M^{\prime \prime}$, and the arguments above imply ${ }^{33}$

$$
\begin{aligned}
& \frac{b_{1}+b_{2}+b_{3}}{a_{1}+a_{2}+a_{3}} \geq \frac{\left|L^{H}\left(A_{1} \cup A_{2} \cup A_{3}\right)\right|}{\left|A_{1} \cup A_{2} \cup A_{3}\right|} \geq r \\
& r=\frac{\left|L^{H}\left(M^{\prime}\right)\right|}{\left|M^{\prime}\right|} \geq \frac{b_{1}+b_{2}+a_{5}}{a_{1}+a_{2}+a_{4}} \\
& r=\frac{\left|L^{H}\left(M^{\prime \prime}\right)\right|}{\left|M^{\prime \prime}\right|} \geq \frac{b_{2}+b_{3}+a_{4}}{a_{2}+a_{3}+a_{5}} \\
& \frac{b_{2}}{a_{2}}=\frac{\left|L^{H}\left(A_{2}\right)\right|}{\left|A_{2}\right|} \geq r,
\end{aligned}
$$

which can be rewritten as

$$
\begin{aligned}
b_{1}+b_{2}+b_{3} & \geq r a_{1}+r a_{2}+r a_{3} \\
r a_{1}+r a_{2}+r a_{4} & \geq b_{1}+b_{2}+a_{5} \\
r a_{2}+r a_{3}+r a_{5} & \geq b_{2}+b_{3}+a_{4} \\
b_{2} & \geq r a_{2} .
\end{aligned}
$$

Adding up all the inequalities above and canceling terms we obtain that

$$
(r-1)\left(a_{4}+a_{5}\right) \geq 0
$$

Since $r<1$, it follows that $a_{4}+a_{5}=0$; hence, there are no $H$ links between $M^{\prime}$ and $M^{\prime \prime}$, so the set $M^{\prime} \cup M^{\prime \prime}$ is $H$-independent. Moreover, $A_{4}=A_{5}=\varnothing$, and thus $M^{\prime} \cup M^{\prime \prime}=A_{1} \cup A_{2} \cup A_{3}$.

\footnotetext{
${ }^{33}$ The case $a_{1}+a_{2}+a_{3}=0$ is not possible, as it would lead to $L^{H}\left(M^{\prime}\right) \supset M^{\prime \prime}$ and $L^{H}\left(M^{\prime \prime}\right) \supset M^{\prime}$, which can hold simultaneously only if $r \geq 1$. If $a_{2}=0$, the bottom inequality becomes irrelevant for the proof.
} 
As the sum of all weak inequalities (17)-(20) leads to an equality, it follows that (13) $-(20)$ hold with equality. In particular,

$$
\frac{b_{1}+b_{2}+b_{3}}{a_{1}+a_{2}+a_{3}}=\frac{\left|L^{H}\left(A_{1} \cup A_{2} \cup A_{3}\right)\right|}{\left|A_{1} \cup A_{2} \cup A_{3}\right|}=r .
$$

Therefore,

$$
\frac{\left|L^{H}\left(M^{\prime} \cup M^{\prime \prime}\right)\right|}{\left|M^{\prime} \cup M^{\prime \prime}\right|}=r
$$

which finishes the proof.

\section{PROOF OF PROPOSITION 3:}

It is sufficient to show that $\left(r_{s}\right)_{s}$ is strictly increasing. We proceed by contradiction. Suppose that $r_{s} \leq r_{s-1}$. Then it must be that $1<s<\bar{s}$.

By Lemma $4, L^{G_{s-1}}\left(M_{s-1} \cup M_{s}\right)=L_{s-1} \cup L_{s}$ and $M_{s-1} \cup M_{s}$ is a $G$-independent set. Since

$$
\frac{\left|L_{s-1}\right|}{\left|M_{s-1}\right|}=r_{s-1} \text { and } \frac{\left|L_{s}\right|}{\left|M_{s}\right|}=r_{s} \leq r_{s-1},
$$

it follows that

$$
\frac{\left|L^{G_{s-1}}\left(M_{s-1} \cup M_{s}\right)\right|}{\left|M_{s-1} \cup M_{s}\right|}=\frac{\left|L_{s-1}\right|+\left|L_{s}\right|}{\left|M_{s-1}\right|+\left|M_{s}\right|} \leq r_{s-1} .
$$

Therefore,

$$
M_{s-1} \cup M_{s} \in \underset{M \subset N_{s-1}, M \in \mathcal{I}(G)}{\arg \min } \frac{\left|L^{G_{s-1}}(M)\right|}{|M|},
$$

a contradiction with $M_{s-1}$ being the union of all the minimizers of the expression above.

PROOF OF THEOREM 4, CASE $s=\bar{s}$ :

This case is relevant only when $N_{\bar{s}} \neq 0$, which is assumed in the claims below. Note that $r_{\bar{s}} \geq 1$.

CLAIM 4.5: $v_{k}^{*} \geq 1 / 2, \forall k \in N_{\bar{s}}$

Again, let $\underline{x}_{\bar{s}}=\min _{i \in N_{\bar{s}}} v_{i}^{*}, \underline{M}_{\bar{s}}=\arg \min _{i \in N_{\bar{s}}} v_{i}^{*}$ and $\underline{L}_{\bar{s}}=L^{G_{\bar{s}}}\left(\underline{M}_{\bar{s}}\right)$. We show that $\underline{x}_{\bar{s}} \geq 1 / 2$ by contradiction. Suppose that $\underline{x}_{\bar{s}}<1 / 2$.

By arguments parallel to those in Claim 4.2, under the assumption that $\underline{x}_{\bar{s}}<1 / 2$, $L^{G^{*}}\left(\underline{L}_{\bar{s}}\right) \subset \underline{M}_{\bar{s}}$ and $\underline{L}_{\bar{s}}$ is $G^{*}$-independent. Theorem 3 implies that

$$
\underline{x}_{\bar{s}}=\max _{i \in L^{G^{*}}\left(\underline{L}_{\bar{s}}\right)} v_{i}^{*} \geq \frac{\left|\underline{L}_{\bar{s}}\right|}{\left|\underline{M}_{\bar{s}}\right|+\left|\underline{L}_{\bar{s}}\right|} .
$$


Since $\underline{x}_{\bar{s}}<1 / 2$ and $\underline{L}_{\bar{s}}=L^{G_{\bar{s}}}\left(\underline{M}_{\bar{s}}\right)$, we obtain that

$$
1>\frac{\left|L^{G_{\bar{s}}}\left(\underline{M}_{\bar{s}}\right)\right|}{\left|\underline{M}_{\bar{s}}\right|},
$$

which is a contradiction with $r_{\bar{s}} \geq 1$.

CLAIM 4.6: $v_{k}^{*} \leq 1 / 2, \forall k \in N_{\bar{s}}$

Fix $k \in N_{\bar{s}}$. By Claim 4.5, $v_{k}^{*} \geq 1 / 2$. One consequence of Lemma 4 is that $k$ has no $G$ links to players in $M_{1} \cup M_{2} \cup \ldots \cup M_{\bar{s}-1}$. By Proposition 2 , as $v_{k}^{*} \geq 1 / 2$, there are no $G^{*}$ links from $k$ to players $j \in L_{1} \cup L_{2} \cup \ldots \cup L_{\bar{s}-1}$, since for these players $v_{j}^{*} \geq 1-x_{\bar{s}-1}>1 / 2$. Therefore, $k$ may only have $G^{*}$ links to players in $N_{\bar{s}}$. But Claim 4.5 showed that the limit equilibrium payoff of every player in $N_{\bar{s}}$ is at least $1 / 2$. Then Proposition 2 and Lemma 1 imply that $v_{k}^{*} \leq 1 / 2$.

Claims 4.5 and 4.6 show that $v_{k}^{*}=1 / 2$ for all $k \in N_{\bar{s}}$.

\section{PROOF OF THEOREM 6:}

Let $G$ be a buyer-seller network. By Proposition $3^{B S}$ and Theorem $4^{B S}, G$ is nondiscriminatory if and only if $B_{1}=B, S_{1}=S, r_{1}=1 / \beta$ if and only if $\left|L^{G}(M)\right| /|M| \geq$ $1 / \beta$ for all $M \subset B$. Let $H$ be the graph obtained from $G$ by replacing each vertex corresponding to a seller with $\beta$ identical copies (each copy is connected to all buyers whom the corresponding seller was connected to). Note that $\left|L^{G}(M)\right| /|M| \geq 1 / \beta$, $\forall M \subset B$ is equivalent to $\left|L^{H}(M)\right| /|M| \geq 1, \forall M \subset B$. Since the numbers of buyers and sellers in $H$ are equal, P. Hall (1935)'s theorem implies that the latter condition is equivalent to $H$ having a perfect matching. By construction, $H$ has a perfect matching if and only if $G$ has an edge cover that is a disjoint union of $\beta$-buyer-seller clusters, which completes the proof.

\section{REFERENCES}

Abreu, Dilip, and Mihai Manea. 2009a. "Bargaining and Efficiency in Networks." http://www. princeton.edu/ dabreu/Bargaining\%20and\%20Efficiency\%20in\%20Networks.pdf.

Abreu, Dilip, and Mihai Manea. 2009b. "Markov Perfect Equilibria in a Model of Bargaining in Networks." http://econ-www.mit.edu/files/6782.

Berge, Claude. 1981. "Some Common Properties for Regularizable Graphs, Edge-Critical Graphs and B-Graphs.” In Graph Theory and Algorithms. Vol. 108, Lecture Notes in Computer Science, ed. N. Saito and T. Nishizeki, 108-23. New York: Springer-Verlag.

-Binmore, K. G., and M. J. Herrero. 1988a. "Matching and Bargaining in Dynamic Markets." Review of Economic Studies, 55(1): 17-31.

Binmore, K. G., and M. J. Herrero. 1988b. "Security Equilibrium." Review of Economic Studies, 55(1): 33-48.

Corominas-Bosch, Margarida. 2004. "Bargaining in a Network of Buyers and Sellers." Journal of Economic Theory, 115(1): 35-77.

Crawford, Vincent P., and Elsie Marie Knoer. 1981. "Job Matching with Heterogeneous Firms and Workers." Econometrica, 49(2): 437-50.

-Gale, Douglas M. 1987. "Limit Theorems for Markets with Sequential Bargaining." Journal of Economic Theory, 43(1): 20-54.

Hall, P. 1935. "On Representatives of Subsets.” Journal of the London Mathematical Society, S1-10(1): 26-30. 
Jackson, Matthew O. 2008. Social and Economic Networks. Princeton, NJ: Princeton University Press.

Kranton, Rachel E., and Deborah F. Minehart. 2001. "A Theory of Buyer-Seller Networks." American Economic Review, 91(3): 485-508.

Manea, Mihai. 2010. "Bargaining in Dynamic Markets with Multiple Populations." http://econ-www. mit.edu/files/6783.

Polanski, Arnold. 2007. "Bilateral Bargaining in Networks." Journal of Economic Theory, 134(1): 557-65.

Rubinstein, Ariel, and Asher Wolinsky. 1985. "Equilibrium in a Market with Sequential Bargaining." Econometrica, 53(5): 1133-50.

Rubinstein, Ariel, and Asher Wolinsky. 1990. "Decentralized Trading, Strategic Behaviour and the Walrasian Outcome." Review of Economic Studies, 57(1): 63-78. 